\title{
Gaia DR2 study of Herbig Ae/Be stars ${ }^{\star}$
}

\author{
M. Vioque ${ }^{1,2}$, R. D. Oudmaijer ${ }^{1}$, D. Baines ${ }^{3}$, I. Mendigutía ${ }^{4}$, and R. Pérez-Martínez ${ }^{2}$
}

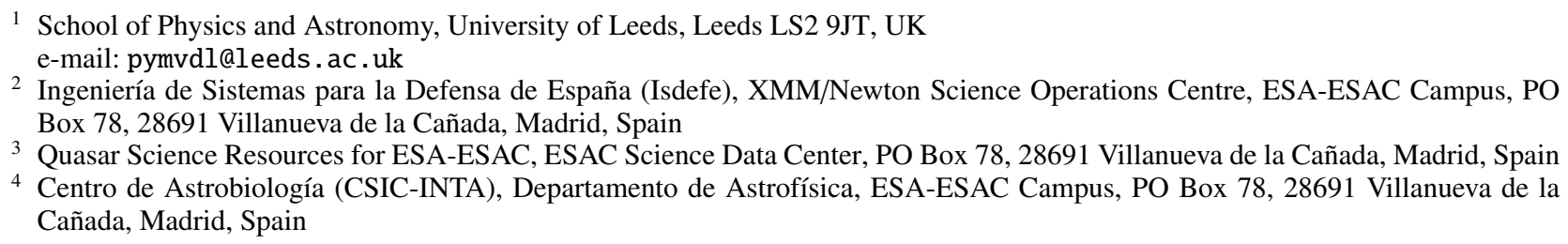

Received 21 February 2018 / Accepted 29 July 2018

\section{ABSTRACT}

\begin{abstract}
Aims. We use Gaia Data Release 2 (DR2) to place 252 Herbig Ae/Be stars in the Hertzsprung-Russell diagram and investigate their characteristics and properties.

Methods. For all known Herbig Ae/Be stars with parallaxes in Gaia DR2, we collected their atmospheric parameters and photometric and extinction values from the literature. To these data we added near-infrared and mid-infrared photometry, and collected $\mathrm{H} \alpha$ emission line properties such as equivalent widths and line profiles, and their binarity status. In addition, we developed a photometric variability indicator from Gaia's DR2 information.

Results. We provide masses, ages, luminosities, distances, photometric variabilities and IR excesses homogeneously derived for the most complete sample of Herbig Ae/Be stars to date. We find that high-mass stars have a much smaller IR excess and have much lower optical variabilities compared to lower-mass stars, with the break at around $7 M_{\odot} . \mathrm{H} \alpha$ emission is generally correlated with IR excess, with the correlation being stronger for IR emission at wavelengths tracing the hot dust closest to the star. The variability indicator as developed by us shows that $\sim 25 \%$ of all Herbig Ae/Be stars are strongly variable. We observe that the strongly variable objects display doubly peaked $\mathrm{H} \alpha$ line profiles, indicating an edge-on disk.

Conclusions. The fraction of strongly variable Herbig Ae stars is close to that found for A-type UX Ori stars. It had been suggested that this variability is in most cases due to asymmetric dusty disk structures seen edge-on. The observation here is in strong support of this hypothesis. Finally, the difference in dust properties occurs at $7 M_{\odot}$, while various properties traced at UV/optical wavelengths differ at a lower mass, $3 M_{\odot}$. The latter has been linked to different accretion mechanisms at work, whereas the differing IR properties and photometric variabilities are related to different or differently acting (dust-)disk-dispersal mechanisms.
\end{abstract}

Key words. stars: variables: T-Tauri - stars: variables: Herbig Ae/Be - Hertzsprung-Russell and C-M diagrams - stars: formation stars: pre-main sequence - stars: emission-line, $\mathrm{Be}$ - infrared: stars

\section{Introduction}

Herbig Ae/Be stars (HAeBes) are pre-main sequence (PMS) stars of intermediate mass, spanning the range between lowmass T-Tauri stars and the embedded massive young stellar objects (MYSOs). They are optically bright so they are much easier to observe and to study than MYSOs and it is expected that within the mass range of HAeBes a change in accretion mechanism occurs, from the magnetically controlled accretion acting for T-Tauri stars (see Bouvier et al. 2007) to a yet unknown mechanism for high-mass stars. Indeed, there is evidence that the magnetically driven accretion model is valid for Herbig Ae stars but not for several Herbig Be stars (Fairlamb et al. 2015; Ababakr et al. 2017; Oudmaijer 2017; Grady et al. 2010; Schöller et al. 2016). Moreover, there are multiple pieces of evidence suggesting that Herbig Ae and T-Tauri stars behave similarly, while Herbig Be stars behave differently. In fact, Herbig Ae and Herbig Be stars have different observational

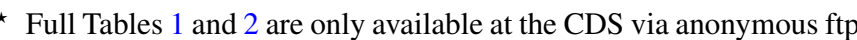
to cdsarc.u-strasbg. fr (130.79.128.5) or via http://cdsarc. u-strasbg.fr/viz-bin/qcat?J/A+A/620/A128
}

properties. Examples of this are the different outer gas dispersal rates (higher for Herbig Be stars, Fuente et al. 1998), the higher incidence of clustering scenarios for Herbig Be stars (Testi et al. 1999), and the evidence of Herbig Be stars hosting denser and larger inner gaseous disks (Ilee et al. 2014; Monnier et al. 2005) which may suggest a different accretion scenario with the disk reaching directly into the star (Kraus 2015). Other spectro-photometric (Mendigutía et al. 2011b; Cauley \& Johns-Krull 2015; Patel et al. 2017) and spectropolarimetric studies (Vink et al. 2002) also point to a change in accretion physics within the Herbig Ae/Be stars mass range. In addition, Herbig Be stars are more likely to be found in binaries than Herbig Ae stars (Baines et al. 2006).

An important indicator of their PMS nature, together with emission lines, is the infrared (IR) excess that also traces the Herbig Ae/Be forming environment. The IR excess profiles have been classified into two groups differentiated by a flat or rising shape of the continuum (Meeus et al. 2001). This difference has a geometric origin depending on the presence of flaring outer disks and puffed-up inner disks (Dullemond \& Dominik 2004a, 2004b, 2005), and the presence of gaps in the disk (Maaskant et al. 2013; Honda et al. 2015). The IR excess of HAeBes is expected to be characteristic and different from the 
IR excess of other similar objects like, for example, ordinary Be stars (Finkenzeller \& Mundt 1984).

Herbig Ae/Be stars are known to present irregular photometric variations, with a typical timescale from days to weeks (Eiroa et al. 2002; Oudmaijer et al. 2001) and of the order of one magnitude in the optical. This variability is typically understood as being due to variable extinction, caused by, example, rotating circumstellar disks, or as an effect of rotation on cold photospheric spots and also pulsation due to the source crossing the instability strip in the HR diagram (Marconi \& Palla 1998). An extreme case of large non-periodic photometric and polarimetric variations is observed in UX Ori type stars (UXORs) with amplitudes up to 2-3 mag. Many of them are catalogued as HAeBes and their extreme variability is explained by eclipsing dust clouds in nearly edge-on sources and the scattering radiation in the circumstellar environment (see Grinin 2000 and references therein; Natta et al. 1997; Natta \& Whitney 2000).

Infrared photometric variability related to disk structure variations is not always correlated with the optical variability (Eiroa et al. 2002) which implies that different mechanisms regarding both the disk structure and accretion underlie the final observed variability. Spectroscopic variability is also present in Herbig Ae/Be stars (Mendigutía et al. 2011a).

With the advent of the second data release of Gaia (DR2, Gaia Collaboration 2016, 2018b) providing parallaxes to over 1.3 billion objects (Lindegren et al. 2018), including the majority of known Herbig Ae/Be stars, the time is right for a new study on the properties of the class. Gaia DR2 contains a fivedimensional astrometric solution $\left(\alpha, \delta, \mu_{\alpha}, \mu_{\delta}\right.$ and parallax $\left.(\varpi)\right)$ up to $G \lesssim 21$ (white $G$ band, described in Evans et al. 2018). Almost all of the known Herbig Ae/Be stars have parallaxes in Gaia DR2, which allowed luminosities to be derived and 252 HAeBes to be placed in the HR diagram, a tenfold increase on earlier studies using Hipparcos data alone.

The paper is organised as follows: in Sect. 2, we describe the data acquisition of not only the parallaxes, but also optical and IR photometry, effective temperatures, extinction values, $\mathrm{H} \alpha$ emission line information, and binarity. In Sect. 3, we derive the stellar luminosities and place the objects in a Hertzsprung-Russell (HR) diagram, while we also present a method to derive a statistical assessment of the objects' variability in Gaia's database. In addition, we homogeneously derive masses and ages for all the sources, together with near-infrared (NIR) and mid-infrared (MIR) excesses. In Sect. 4, we carry out an analysis of the data and present various correlations and interdependencies, which we discuss in the context of intermediate-mass star formation in Sect. 5. We conclude in Sect. 6.

\section{Data acquisition}

\subsection{Construction of the sample}

We have gathered the majority of Herbig Ae/Be stars known and proposed to date from different works (272, see Chen et al. 2016 for a compilation of most of them). Chen et al. (2016) based their sample mostly on the work of Zhang et al. (2006) which in turn is based on the work of Thé et al. (1994) and Vieira et al. (2003). In addition, we included a few HAeBes from Alecian et al. (2013), Baines et al. (2006), Carmona et al. (2010), Fairlamb et al. (2015), Hernández et al. (2005), Manoj et al. (2006) and Sartori et al. (2010) that are not present in the aforementioned papers.

Although Herbig Ae/Be stars have long been considered, by definition, to be type $\mathrm{A}$ or $\mathrm{B}$, there should be some flexibility in this constraint as the physical boundary between Herbig Ae stars and intermediate-mass T-Tauris is relatively poorly studied. This is because spectral types of T-Tauri stars are typically K-M with some G-type objects, while Herbig Ae/Be stars are, quite unsurprisingly, limited to A and B spectral type. Therefore, PMS stars of intermediate spectral types have often been largely understudied. We therefore keep objects with F-type classification in Chen et al. (2016) in the sample. Similarly, no upper limit in mass was imposed, leaving the separation between MYSOs and HAeBes to the optical brightness of the sources ${ }^{1}$.

Subsequently, we cross-matched the sources with Gaia DR2. Detections were considered to be matched with the catalogue when their coordinates agreed to within 0.5 arcsec. If more than one match was found, we took the closest one. If no match was found within 0.5 arcsec, successive cross-matches with larger apertures were performed up to 2 arcsec. In these latter cases an individual inspection of the cross-match was applied. Finally, a comparison between the Johnson $V$ band magnitudes and the Gaia filters was made for each source in order to discard possible incorrect matches. This provided us with parallaxes for $254 \mathrm{HAeBes}$.

As Lindegren et al. (2018) point out, not all Gaia DR2 parallaxes are of the same quality, and some values - despite their sometimes very small error bars - appear erroneous (e.g. Lindegren et al. 2018). We included the following constraint in astrometric quality following the indications in Appendix C of Lindegren et al. (2018) and what was applied in Gaia Collaboration (2018a). This constraint will remove objects with spurious parallaxes from the sample:

$u<1.2 \times \max \left(1, \mathrm{e}^{-0.2(G-19.5)}\right)$

where $G$ is the Gaia $G$ band and $u$ is the unit weight error, defined as the square root of the ratio of the astrometric_chi2_al and (astrometric_n_good_obs_al - 5) columns (Lindegren et al. 2018, their Eq. (C.2)). Of our sources, 228 satisfy this condition.

Some objects are found to be very close to this condition; PDS 144S, PV Cep and V892 Tau, and as we show below, they would appear significantly below the MS in the HR-diagram. Given that the Lindegren condition is presented as a guideline rather than a rule by the Gaia astrometry team, we decided to treat these three objects as if they satisfy Eq. (1) as well.

We refer to the set of astrometrically well-behaved sources as the high-quality sample and to those that do not satisfy Eq. (1) as the low-quality sample. We are not able to place two sources in the HR diagram due to a lack of appropriate parameters (Sect. 2.2). In addition, we move five more sources to the lowquality sample in Sect. 3.1 for different reasons. Summarising, there are 218 objects $(228-3-2-5)$ in the final high-quality sample and 34 in the low-quality one. Information about the objects in different samples is presented in separated tables (Tables 1 and 2 , available in their entirety at the CDS). The high-quality sample will be the one taken into account in further considerations unless otherwise specified.

Distances are not obtained by straightforwardly inverting the parallax. The conversion of one parameter to the other one is not strictly trivial because of the non-linearity of the inverse function (see for example Bailer-Jones 2015). In the case of Gaia DR2, Bailer-Jones et al. (2018) proposed distance values using a weak distance prior that follows a galactic model. Their

\footnotetext{
1 The MYSOs are typically IR-bright and optically faint (Lumsden et al. 2013). However, a number of optically visible objects are known to have passed all selection criteria such as the early-type objects PDS 27 and PDS 37 that are also classified as Herbig Be stars (Ababakr et al. 2015).
} 
distances begin to differ from the distances obtained through simple inversion for sources with large errors, $\sigma_{\varpi} / \varpi \gtrsim 0.5$. Therefore, in our initial (high- and low-quality) sample only a small subset of 12 Herbig Ae/Be stars suffers substantially from this effect. Following the indications in Luri et al. (2018) on how to treat the Gaia parallaxes, we decided to apply a simpler exponentially decreasing prior to estimate distances. For completeness, we should note that the parallaxes provided by Gaia DR2 have a regional and not Gaussian systematic error as large as 0.1 mas and a global zero point error of about -0.029 mas which are not included in the Gaussian random errors provided in the Gaia archive (see Arenou et al. 2018; Lindegren et al. 2018). The uncertainty in the parallaxes is therefore slightly underestimated. The final errors in the high-quality sample range from 0.016 to 0.37 mas.

Herbig Ae/Be stars have been historically confused with classical Be stars, with which they share many characteristics (Rivinius et al. 2013; Klement et al. 2017; Grundstrom \& Gies 2006). Indeed, the nature of some of the objects in our sample is still under debate. An interesting example in this respect is HD 76534, a B2Ve object that appears in listings of Be stars (e.g. Oudmaijer \& Drew 1997) and Herbig Be stars alike (Fairlamb et al. 2015). The latest dedicated study puts the object in the Herbig Be category (Patel et al. 2017). To assess the effect of ambiguous classifications in our study, next to the full sample, we also consider the subset of Herbig Ae/Be stars in Table 1 of Thé et al. (1994). This catalogue contains all historically known, and best studied, Herbig Ae/Be stars. Ninety-eight of our 254 initial sources with parallaxes are present in this table (their best candidates). This Thé et al. (1994) subset is indicated in Tables 1 and 2 for the high- and low-quality samples, respectively.

\subsection{Atmospheric parameters, photometry, and extinction values}

We obtained atmospheric parameters and photometric and extinction values for all the sources from the literature. These were mainly Alecian et al. (2013), Carmona et al. (2010), Chen et al. (2016), Fairlamb et al. (2015), Hernández et al. (2004, 2005), Manoj et al. (2006), Montesinos et al. (2009), Mendigutía et al. (2012), Sartori et al. (2010), Vieira et al. (2003) and the APASS Data Release 9. Whenever the effective temperature ( $\left.T_{\text {eff }}\right)$ was not available it was derived from the spectral type with the effective temperature calibration tables of Gray \& Corbally (2009). An uncertainty of 1 sub-spectral type was assigned in all cases. When not listed in the literature, $A_{\mathrm{V}}$ values were derived from the observed photometry and using the intrinsic colours of Pecaut \& Mamajek (2013). An $R_{\mathrm{V}}=3.1$ was used in all cases in which $A_{\mathrm{V}}$ was derived; although other studies like Hernández et al. (2004) or Manoj et al. (2006) have suggested that a larger value of for example $R_{\mathrm{V}}=5$ could be more appropriate for HAeBes where local extinction dominates the total extinction. This is a topic for future investigations using diffuse interstellar bands (as done by e.g. Oudmaijer et al. 1997). The relevant data of each source is presented in Tables 1 and 2 for the high- and low-quality samples, respectively.

HAeBes usually show photometric variability. Thus, for objects with multi-epoch photometry available, we selected the brightest set to determine the extinction towards the objects and thus their intrinsic brightnesses. As we also show below, the variability is often caused by irregular extinction; using those data with minimum extinction introduces the smallest errors in the determination of the stellar parameters. For this reason, we only used simultaneous photometry when deriving $A_{\mathrm{V}}$ values. All the photometric values were corrected for extinction using the reddening law of Cardelli et al. (1989).

Two sources, V833 Ori and GSC 1829-0331, do not have enough simultaneous photometry available to derive extinctions for them and therefore they were excluded for the sample. The total number of Herbig $\mathrm{Ae} / \mathrm{Be}$ stars that can be placed in the HR diagram and for which we can derive stellar luminosities, masses, ages, IR excesses, and variabilities in Sect. 3 is therefore reduced to 252 objects.

\subsection{Infrared photometry}

All the sources were cross-matched with the Two-Micron All Sky Survey (2MASS, see Skrutskie et al. 2006) and with the Wide-Field Infrared Survey Explorer "AllWISE" all-sky catalogue (hereafter WISE, see Cutri et al. 2013). Both of these surveys contain hundreds of millions of stars, guaranteeing a large overlap with Gaia. We used a three-arcsecond aperture for the cross-match. The few sources that did not lie within that threearcsecond threshold were studied individually and, if present, their IR photometry was included. This provides values and uncertainties for the $J, H$, and $K_{\mathrm{s}}$ bands $(1.24,1.66$, and $2.16 \mu \mathrm{m}$, respectively) and for the $W 1, W 2, W 3$, and $W 4$ bands (3.4, 4.6, 12 , and $22 \mu \mathrm{m}$ respectively) for most of the HAeBes. We note that for some sources some of the bands may be missing or may just be upper limits. We double-checked all IR matches with the dereddened optical photometry and found no inconsistencies.

\subsection{Ho equivalent width and emission line profile}

We collected all the $\mathrm{H} \alpha$ equivalent widths (EW) we could find in the literature for the Herbig Ae/Be stars. Not only the intensity of the line but also the shape contains very useful information. Therefore, when possible, information about the shape of the $\mathrm{H} \alpha$ line was included. We have classified the $\mathrm{H} \alpha$ line profile as single-peaked (s), double-peaked (d) and showing a P-Cygni profile $(\mathrm{P})$, both regular or inverse. Equivalent width and line shape information are presented in Tables 1 and 2 for the high- and low-quality samples, respectively. Many Herbig Ae/Be stars are quite variable in their $\mathrm{H} \alpha$ emission and their EWs may significantly change on short timescales (e.g. Costigan et al. 2014). This is also the case for the line shape, although spot checks on objects that have more than one $\mathrm{H} \alpha$ observation listed in the literature appear to indicate that there are not many changes in line profile classification (see also e.g. Aarnio et al. 2017), although changes between single-peaked and double-peaked profiles in a given star are also observed (Mendigutía et al. 2011a). We do note the additional complication that emission line shapes are often difficult to unambiguously classify.

Regarding the $\mathrm{H} \alpha$ EWs compiled, we note that our main references (Fairlamb et al. 2015; Mendigutía et al. 2011a) provide the non-photospheric contribution of the $\mathrm{EW}$, while most other authors state the observed EW, which includes the photospheric contribution. This photospheric absorption peaks for A0-A1 type objects, with EW values of $\sim+10 \AA$ (see e.g. Fig. 7 of Joner \& Hintz 2015) but is only $\sim+2 \AA$ for B0 objects. We used the Joner \& Hintz (2015) results to correct those EWs that were not corrected for absorption.

We have $\mathrm{H} \alpha$ EWs for 218 of the 252 HAeBes and line profiles for 197 of these: $31 \%$ are single-peaked, $52 \%$ are double-peaked, and $17 \%$ are P-Cygni (of which the vast majority are of regular P-Cygni type). This is in agreement with Finkenzeller \& Mundt (1984) who found that out of 57 HAeBes, $25 \%$ were singlepeaked, $50 \%$ showed double-peaked $\mathrm{H} \alpha$ profiles, and $20 \%$ 


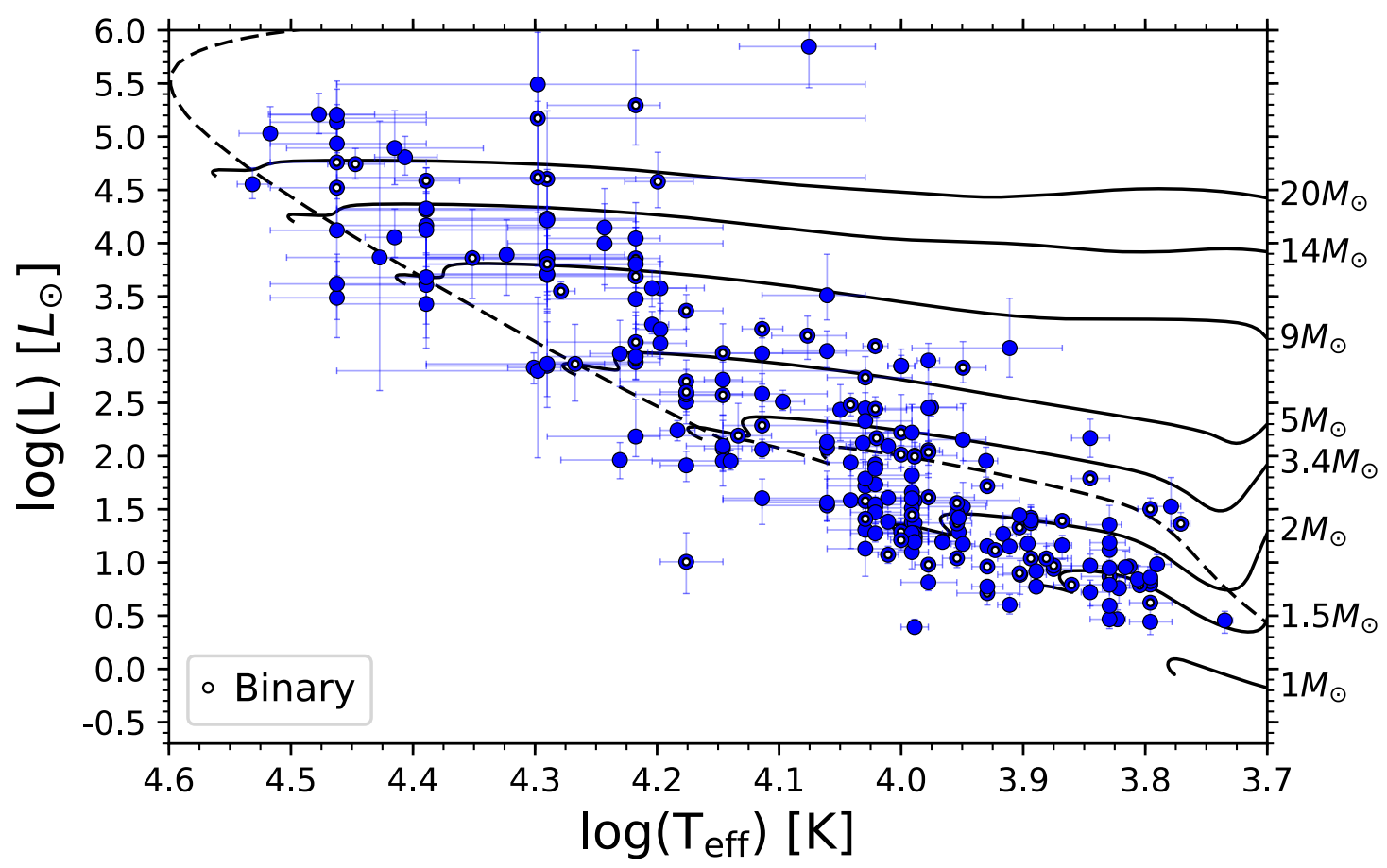

Fig. 1. 223 Herbig Ae/Be stars in the HR diagram satisfying Eq. (1) constraint. In most cases vertical error bars are dominated by parallax uncertainties. Sources with a white dot have been classified as binaries. The mass of each PMS track (Bressan et al. 2012) is indicated on the righthand side. An isochrone (Bressan et al. 2012; Marigo et al. 2017) of 2.5 Myr is also shown for reference as a dashed line.
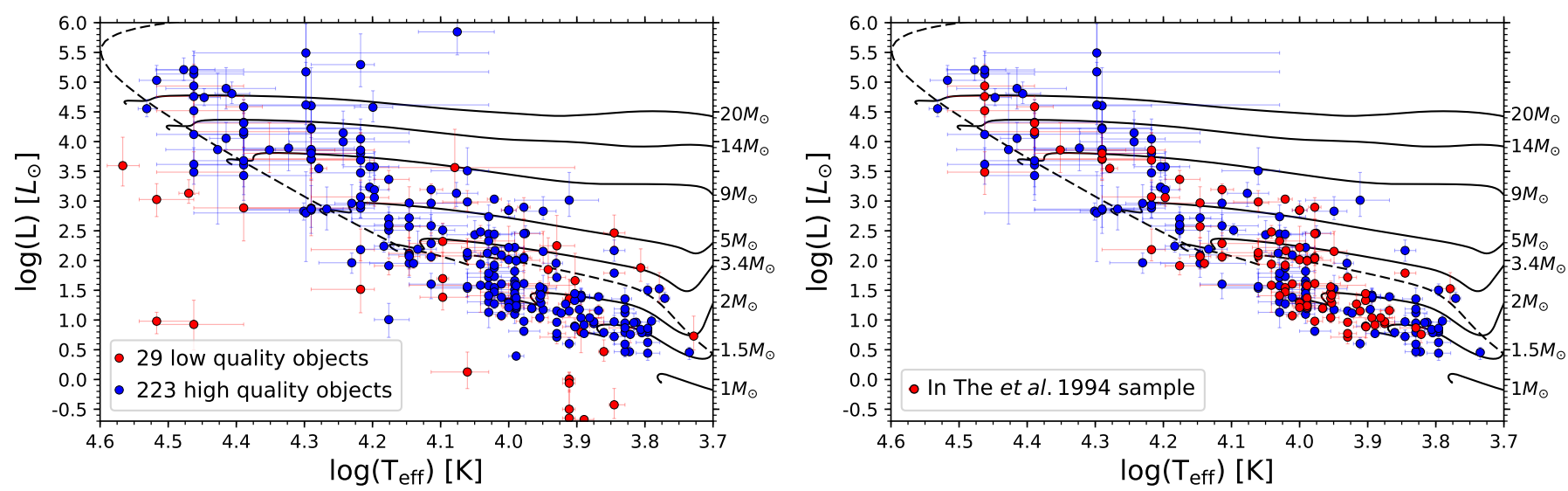

Fig. 2. Left panel: 223 high-quality and 29 low-quality Herbig Ae/Be stars in the HR diagram after the cut in astrometric quality described in Eq. (1). Right panel: $218 \mathrm{Herbig} \mathrm{Ae/Be} \mathrm{stars} \mathrm{in} \mathrm{the} \mathrm{final} \mathrm{high-quality} \mathrm{sample} \mathrm{after} \mathrm{removing} \mathrm{the} \mathrm{five} \mathrm{problematic} \mathrm{objects} \mathrm{described} \mathrm{in} \mathrm{Sect.} \mathrm{3.1.}$ Those objects present in Table 1 of The et al. (1994) are shown in red. The mass of each PMS track (Bressan et al. 2012) is indicated on the right-hand side. An isochrone (Bressan et al. 2012; Marigo et al. 2017) of 2.5 Myr is also shown for reference as a dashed line.

presented a P-Cygni profile (both regular and inverse). The main references for the EW values are Baines et al. (2006), Fairlamb et al. (2017), Hernández et al. (2004), Mendigutía et al. (2011a) and Wheelwright et al. (2010). The main references for the line profiles are van den Ancker et al. (1998), Baines et al. (2006), Mendigutía et al. (2011a), Vieira et al. (2003) and Wheelwright et al. (2010). The rest of the references can be found in Tables 1 and 2.

\subsection{Binarity}

More than half of the Herbig Ae/Be stars are known to be in binary systems (Duchêne 2015). The true number is likely much larger, as there have been a small number of targeted surveys for binarity of HAeBe stars, the largest are Wheelwright et al. (2010) and Baines et al. (2006) who performed spectroastrometry of 45 HAeBes and 31 HAeBes, respectively, probing companions in the $\approx 0.1-2$ arcsec range, and Leinert et al. (1997) who performed speckle interferometry of 31 objects, sampling separations of order 0.1 arcsec. Eighty-one of the 252 HAeBes $(\sim 32 \%)$ of our set are catalogued as binary systems, a fraction that is in agreement with the Duchêne (2015) findings if we take into account the large number of faint Herbig Ae/Be stars which have never been studied for binarity. The binary status of each $\mathrm{HAeBe}$ is presented in Tables 1 and 2 for the high- and lowquality samples, respectively; main references were Baines et al. (2006), Leinert et al. (1997) and Wheelwright et al. (2010); we refer to Tables 1 and 2 for a complete list. 


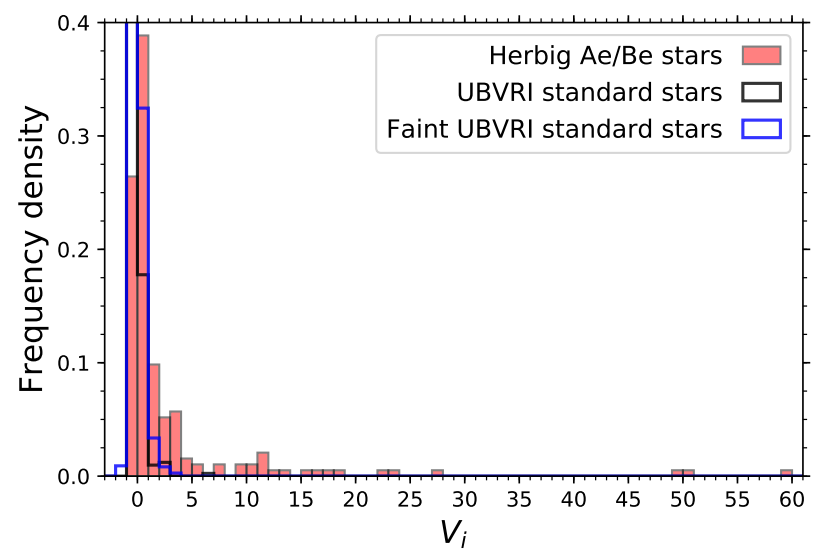

Fig. 3. Distribution of the variability indicator for Herbig Ae/Be stars and two catalogues of photometric standards; one of bright sources (Landolt 2009) and one of faint sources (Clem \& Landolt 2016). As a class, the Herbig Ae/Be stars are more variable than the photometric standards.

Baines et al. (2006) found a typical wide (few hundred au) separation in the binary systems. Wheelwright et al. (2010) detected no binaries closer than 30 au and established a range of $\approx 40-4000$ au in their data.

\section{Derived quantities}

\subsection{Luminosity and Hertzsprung-Russell diagram}

Using the parallaxes, atmospheric parameters, and extinction values, we derived the luminosity for the $252 \mathrm{HAeBes}$ with parallaxes employing a similar method to Fairlamb et al. (2015), which is similar to that of Montesinos et al. (2009) and van den Ancker et al. (1997). In short, it first consists of using values of $T_{\text {eff }}$ and surface gravity $(\log (g))$ to select an atmosphere model from Castelli \& Kurucz (2004) (referred to as CK-models hereafter) for each star to be used for its intrinsic spectral energy distribution (SED). Solar metallicity CK-models were used in all cases but for BF Ori, RR Tau, SV Cep, XY Per and WW Vul for which the metallicities are known not to be solar from the spectroscopic work of Montesinos et al. (2009). When possible, the $\log (g)$ values were estimated from the luminosity class; otherwise they were taken as 4.00 (typical values range from 3.5 to 4.5). Uncertainties in $\log (g)$ and metallicity can be neglected in our study as their effect on the model SED and derived quantities is negligible.

We then scaled the model to the dereddened photometric Johnson $V$ band. The energy distribution is then integrated over frequency to get the total flux. The final luminosities, presented in Tables 1 and 2 for the high-quality and low-quality samples, respectively, are then obtained by means of the total flux and the parallax. All sources of uncertainty were taken into account at this step including using different $\mathrm{CK}$ models for the different temperatures within the $T_{\text {eff }}$ uncertainty range.

The 223 Herbig Ae/Be stars satisfying the Eq. (1) constraint are plotted in the resulting HR diagram in Fig. 1. This number is an increase of more than a factor of ten compared to the previous, Hipparcos-based study by van den Ancker et al. (1998). Premain sequence evolutionary tracks from Bressan et al. (2012) are also plotted in Fig. 1 in addition to a $2.5 \mathrm{Myr}$ isochrone (Bressan et al. 2012; Marigo et al. 2017), all of them with solar metallicities $(Z=0.01$ and $Y=0.267)$.
Before we analyse this sample, we also plot the HR diagram for all 252 objects with parallaxes (the high- and lowquality samples together, hence including those which failed the Lindegren quality selection criteria) in Fig. 2 on the left. Many of these astrometrically badly behaved sources are located in unphysical positions, significantly below the MS, validating our approach of removing those from our analyses.

Returning to the HR diagram in Fig. 1, there are still several outliers that do not seem to be PMS objects. GSC 5360-1033 and UY Ori appear way below the MS, just like the lower-quality objects that were removed earlier. However, the Gaia DR2 data of these two objects appear to be of good quality.

Regarding GSC 5360-1033, the situation is unclear, an ambiguous spectral type or photometry for this object or an incorrect estimation of the extinction may be the reason for the unexpected location of the object. For UY Ori, Fairlamb et al. 2015 assigned a spectral type of B9, but the photometry listed in SIMBAD indicates a large variability. Pending more certainty, we decided to move these two objects from the high- to the lowquality sample.

MWC 314, MWC 623 and MWC 930 on the other hand appear quite luminous and very much to the right of the MS, something very unusual for high-mass PMS objects. An individual inspection reveals that these objects are more likely to be evolved giants and they appear in the literature as such (e.g. for MWC 314: Carmona et al. 2010, for MWC 623: Lee et al. 2016, for MWC 930: Miroshnichenko et al. 2014). Deciding on the nature of the various Herbig $\mathrm{Ae} / \mathrm{Be}$ candidates in our master sample is not our intention and is beyond the scope of this paper, which is essentially a statistical study. However, these objects occupy a special place in the HR diagram that is consistent with both a pre- and a post-MS nature, while there is much information regarding these objects supporting their post-MS nature. We therefore decided to err on the side of caution and exclude these from further analysis as well.

The final HR diagram without these $2+3$ problematic objects is presented in Fig. 2 on the right. In addition, in this graph, we highlight the sample of The et al. (1994) bonafide HAeBes in red. This final high-quality sample of 218 objects is the one we use in the following plots and studies. The information concerning the 34 discarded objects in the low-quality sample can be found in Table 2 .

In this last high-quality HR diagram we see that there are many more low-mass HAeBes than high-mass HAeBes (69\% of the sources are below $\left.4 M_{\odot}\right)$. This is most likely because of the initial mass function (IMF). This trend of more objects for lower masses discontinues below $\sim 2 M_{\odot}$. This is roughly the mass corresponding to the boundary between MS A- and F-type stars, and thus the traditional lower-mass boundary at which the Herbig $\mathrm{Ae} / \mathrm{Be}$ stars were originally selected.

For lower masses, the sources show a greater spread in temperature, occupying larger parts of the PMS tracks, while, instead, the high-mass objects tend to be predominately located close to the zero-age main sequence (ZAMS). This is likely because the higher the mass, the faster the PMS evolution. This fast evolution could explain why high-mass objects at low temperatures (and thus low surface gravities) are barely present in Fig. 1 or the sample.

We encounter more examples below where high- and lowmass objects have different properties. 
Table 1. Main parameters and IR excess at each bandpass (defined as $F_{\text {observed }} / F_{\mathrm{CK}}$ ) of each Herbig Ae/Be star belonging to the high-quality sample of 218 sources.

\begin{tabular}{|c|c|c|c|c|c|c|c|c|c|}
\hline Name & $\begin{array}{r}\text { RA } \\
\text { (h:m:s) }\end{array}$ & $\begin{array}{r}\text { Dec } \\
\text { (deg:m:s) }\end{array}$ & $\begin{array}{r}\text { Parallax } \\
(\text { mas })\end{array}$ & $\begin{array}{r}\text { Distance } \\
(\mathrm{pc})\end{array}$ & $\begin{array}{l}T_{\text {eff }} \\
(\mathrm{K})\end{array}$ & $\begin{array}{r}\log (L) \\
\left(L_{\odot}\right)\end{array}$ & $\begin{array}{r}A_{\mathrm{V}} \\
(\mathrm{mag})\end{array}$ & $\begin{array}{r}V \\
(\mathrm{mag})\end{array}$ & Binary \\
\hline AB Aur & $04: 55: 45.9$ & $+30: 33: 04$ & $6.140 \pm 0.057$ & $162.9_{-2.4}^{+2.6}$ & $9500_{-790}^{+750}$ & $1.61_{-0.21}^{+0.19}$ & $0.43_{-0.35}^{+0.28}$ & 7.32 & Yes $^{1}$ \\
\hline AK Sco & $16: 54: 44.8$ & $-36: 53: 19$ & $7.113 \pm 0.062$ & $140.6_{-2.0}^{+2.1}$ & $6250_{-250}^{+250}$ & $0.623_{-0.005}^{+0.028}$ & $0.000_{-0.000}^{+0.050}$ & 8.90 & Yes $^{6}$ \\
\hline AS 310 & $18: 33: 21.2$ & $-04: 58: 06$ & $0.390 \pm 0.046$ & $2110_{-240}^{+350}$ & $24500_{-5000}^{+4500}$ & $4.17_{-0.44}^{+0.39}$ & $4.13_{-0.29}^{+0.26}$ & 12.49 & - \\
\hline AS 470 & $21: 36: 14.2$ & $+57: 21: 31$ & $0.137 \pm 0.027$ & $4040_{-440}^{+620}$ & $8200_{-800}^{+1600}$ & $3.01_{-0.27}^{+0.44}$ & $2.27_{-0.42}^{+0.62}$ & 12.44 & - \\
\hline AS 477 & $21: 52: 34.1$ & $+47: 13: 44$ & $1.290 \pm 0.029$ & $773_{-27}^{+30}$ & $10000_{-500}^{+1700}$ & $2.22_{-0.12}^{+0.36}$ & $1.19_{-0.12}^{+0.46}$ & 10.05 & $\mathrm{Yes}^{4}$ \\
\hline$\ldots$ & $\ldots$ & $\ldots$ & $\ldots$ & $\ldots$ & $\ldots$ & $\ldots$ & $\ldots$ & $\ldots$ & $\ldots$ \\
\hline
\end{tabular}

\begin{tabular}{rrrrrrrr}
\hline \hline $\begin{array}{r}\text { Near IR excess } \\
(1.24-3.4 \mu \mathrm{m})\end{array}$ & $\begin{array}{r}\text { Mid IR excess } \\
(3.4-22 \mu \mathrm{m})\end{array}$ & $\begin{array}{r}\mathrm{H} \alpha \mathrm{EW} \\
(\AA)\end{array}$ & $\begin{array}{r}\mathrm{H} \alpha \\
\text { line shape }\end{array}$ & $V_{i}$ & UXOR & $\begin{array}{r}\text { Mass } \\
\left(M_{\odot}\right)\end{array}$ & $\begin{array}{r}\text { Age } \\
(\mathrm{Myr})\end{array}$ \\
\hline $0.32_{-0.13}^{+0.25}$ & $0.27_{-0.12}^{+0.25}$ & $-55.7 \pm 4.5^{6}$ & $\mathrm{P}^{6}$ & 0.21 & - & $2.15_{-0.21}^{+0.36}$ & $4.0_{-1.5}^{+1.4}$ \\
$0.212_{-0.053}^{+0.035}$ & $0.196_{-0.024}^{+0.016}$ & $-5.9 \pm 1.1^{1}$ & $\mathrm{~d}^{28}$ & 12.47 & - & $1.401_{-0.070}^{+0.070}$ & $8.4_{-0.4}^{+1.7}$ \\
- & - & $-8.89 \pm 0.38^{5}$ & $\mathrm{~d}^{26}$ & -0.21 & - & $11.9_{-3.4}^{+4.8}$ & $0.06_{-0.04}^{+0.54}$ \\
$0.031_{-0.031}^{+0.054}$ & $\left(0.95_{-0.82}^{+0.73}\right) \cdot 10^{-2}$ & $-49.2 \pm 4.0^{23}$ & - & 1.22 & - & $7.0_{-1.8}^{+2.8}$ & $0.10_{-0.07}^{+0.20}$ \\
$0.19_{-0.11}^{+0.07}$ & $0.162_{-0.092}^{+0.056}$ & $-38.8 \pm 1.5^{22}$ & $\mathrm{P}^{22}$ & 0.24 & - & $3.3_{-0.4}^{+1.1}$ & $1.25_{-0.73}^{+0.64}$ \\
$\ldots$ & $\ldots$ & $\ldots$ & $\ldots$ & $\ldots$ & $\ldots$ & $\ldots$ & $\ldots$ \\
\hline
\end{tabular}

\begin{tabular}{rrrrrrrr}
\hline \hline$J$ excess & $H$ excess & $K_{\text {s excess }}$ & W excess & $W 2$ excess & $W 3$ excess & W4 excess & The \\
$1.24 \mu \mathrm{m}$ & $1.66 \mu \mathrm{m}$ & $2.16 \mu \mathrm{m}$ & $3.4 \mu \mathrm{m}$ & $4.6 \mu \mathrm{m}$ & $12 \mu \mathrm{m}$ & $22 \mu \mathrm{m}$ \\
\hline 2.79 & 6.01 & 13.23 & 28.77 & - & 240.49 & 2244.34 & Yes \\
1.34 & 1.88 & 3.14 & 7.78 & 11.61 & 49.83 & 400.83 & - \\
0.68 & 0.71 & 0.81 & - & - & - & - & Yes \\
1.21 & 1.28 & 1.71 & 2.15 & 2.77 & 3.21 & 5.36 & - \\
2.00 & - & - & 27.30 & 59.04 & 104.62 & 475.86 & $\ldots$ \\
$\ldots$ & $\ldots$ & $\ldots$ & $\ldots$ & $\ldots$ & $\ldots$ & $\ldots$
\end{tabular}

Notes. This table is available in its entirety at the CDS. A portion is shown here for guidance regarding its form and content. Atmospheric parameters $T_{\text {eff }}, A_{\mathrm{V}}$ and $\mathrm{V}$ taken from the following sources in order of choice: Fairlamb et al. (2015), Montesinos et al. (2009), Hernández et al. (2004), Mendigutía et al. (2012), Carmona et al. (2010), Chen et al. (2016), Alecian et al. (2013), Sartori et al. (2010), Manoj et al. (2006), Hernández et al. (2005) and Vieira et al. (2003), APASS Data Release 9 and the SIMBAD database. If not available they were derived as described in Sect. 2.2. See Sect. 3 for derivation of $L$, Mass and Age. The $\mathrm{H} \alpha$ line profile classification is as follows: single-peaked (s), double-peaked (d) and showing a P-Cygni profile $(\mathrm{P})$, both regular or inverse.

References. The references for binarity are: (1) Baines et al. (2006), (2) Wheelwright et al. (2010), (3) Leinert et al. (1997), (4) Maheswar et al. (2002), (5) Wheelwright et al. (2011), (6) Alecian et al. (2013), (7) Hamaguchi et al. (2008), (8) Dunhill et al. (2015), (9) Coulson \& Walther (1995), (10) Liu et al. (2000), (11) Biller et al. (2012), (12) Schütz et al. (2011), (13) Boersma et al. (2009), (14) Malkov et al. (2006), (15) Arellano Ferro \& Giridhar (2003), (16) Kubát et al. (2010), (17) Morrell \& Levato (1991), (18) Lazareff et al. (2017), (19) Mayer et al. (2016), (20) Folsom et al. (2008), (21) Corporon \& Lagrange (1999), (22) Doering \& Meixner (2009), (23) Chelli et al. (1995), (24) Miroshnichenko et al. (2002), (25) Friedemann et al. (1996), (26) Kraus et al. (2012), (27) Torres et al. (2000), (28) Aspin (1998), (29) Connelley et al. (2008), (30) Millour et al. (2009), (31) Frasca et al. (2016), (32) Marston \& McCollum (2008), (33) Zhang et al. (2017). References for EW values and line shapes: (1) Fairlamb et al. (2017), (2) Carmona et al. (2010), (3) Mendigutía et al. (2011a), (4) Ababakr et al. (2016), (5) Hernández et al. (2004), (6) Baines et al. (2006), (7) Wheelwright et al. (2010), (8) van den Ancker et al. (1998), (9) Oudmaijer \& Drew (1999), (10) Kučerová et al. (2013), (11) Hernández et al. (2005), (12) Dunkin et al. (1997), (13) Pogodin et al. (2012), (14) Miroshnichenko et al. (1999), (15) Sartori et al. (2010), (16) Polster et al. (2012), (17) Manoj et al. (2006), (18) Miroshnichenko et al. (2004), (19) Miroshnichenko et al. (2002), (20) Borges Fernandes et al. (2007), (21) Vieira et al. (2003), (22) Boehm \& Catala (1995), (23) Nakano et al. (2012), (24) Spezzi et al. (2008), (25) Hou et al. (2016), (26) Grinin \& Rostopchina (1996), (27) Vieira et al. (2011), (28) Acke et al. (2005), (29) Herbig \& Bell (1988), (30) Oudmaijer et al. (1998), (31) X-Shooter spectra, 2015, priv. comm., from ESO observing program 084.C-0952A; (32) Ababakr et al. (2017), (33) Zuckerman et al. (2008), (34) Frasca et al. (2016), (35) Miroshnichenko et al. (1998), (36) Miroshnichenko et al. (2000).

\subsection{Mass and age}

Using the isochrones, the masses and ages of the Herbig Ae/Be stars were estimated. We used 100 PARSEC (PAdova and TRieste Stellar Evolution Code) isochrones with solar metallicity (Bressan et al. 2012; Marigo et al. 2017) from 0.01 to $20 \mathrm{Myr}$, from which we only use the PMS tracks. To each Herbig Ae/Be star we assigned the closest two isochrone points in the HR diagram; the solar metallicity isochrones did not match seven sources from the high-quality sample in the HR diagram and isochrones with lower metallicities were used in those cases. As each point is associated with a mass $(M)$ and an age, for each HAeBe we computed an average of those values weighted by the distance to 
Table 2. Main parameters and IR excess at each bandpass (defined as $F_{\text {observed }} / F_{\mathrm{CK}}$ ) of each Herbig Ae/Be star belonging to the low-quality sample of 34 sources.

\begin{tabular}{|c|c|c|c|c|c|c|c|c|c|}
\hline Name & $\begin{array}{r}\text { RA } \\
\text { (h:m:s) }\end{array}$ & $\begin{array}{r}\text { Dec } \\
\text { (deg:m:s) }\end{array}$ & $\begin{array}{r}\text { Parallax } \\
\text { (mas) }\end{array}$ & $\begin{array}{r}\text { Distance } \\
(\mathrm{pc})\end{array}$ & $\begin{array}{l}T_{\text {eff }} \\
(\mathrm{K}) \\
\end{array}$ & $\begin{array}{r}\log (L) \\
\left(L_{\odot}\right)\end{array}$ & $\begin{array}{r}A_{\mathrm{V}} \\
(\mathrm{mag})\end{array}$ & $\begin{array}{r}V \\
\text { (mag) }\end{array}$ & Binary \\
\hline BP Psc & $23: 22: 24.7$ & $-02: 13: 42$ & $2.79 \pm 0.39$ & $350_{-50}^{+110}$ & $5350_{-70}^{+80}$ & $0.73_{-0.23}^{+0.34}$ & $0.83_{-0.24}^{+0.25}$ & 11.53 & - \\
\hline DK Cha & $12: 53: 17.1$ & $-77: 07: 11$ & $4.10 \pm 0.37$ & $243_{-28}^{+47}$ & $7250_{-130}^{+130}$ & $0.47_{-0.16}^{+0.20}$ & $8.12_{-0.14}^{+0.11}$ & 18.54 & - \\
\hline GSC 5360-1033 & $05: 57: 49.5$ & $-14: 05: 34$ & $1.649 \pm 0.034$ & $605_{-19}^{+22}$ & $15000_{-1000}^{+800}$ & $1.01_{-0.30}^{+0.27}$ & $1.60_{-0.50}^{+0.50}$ & 13.91 & Yes $^{29}$ \\
\hline GSC 5988-2257 & $07: 41: 41.1$ & $-20: 00: 13$ & $-4.66 \pm 0.86$ & $980_{-270}^{+520}$ & $16500_{-800}^{+3000}$ & $1.52_{-0.39}^{+0.63}$ & $3.18_{-0.15}^{+0.23}$ & 15.52 & - \\
\hline GSC 6542-2339 & $07: 24: 37.0$ & $-24: 34: 47$ & $1.12 \pm 0.11$ & $850_{-100}^{+160}$ & $32900_{-3900}^{+2000}$ & $3.03_{-0.29}^{+0.27}$ & $5.24_{-0.18}^{+0.14}$ & 15.12 & - \\
\hline$\ldots$ & $\ldots$ & $\ldots$ & $\ldots$ & $\ldots$ & $\ldots$ & $\ldots$ & $\ldots$ & $\ldots$ & $\ldots$ \\
\hline
\end{tabular}

\begin{tabular}{|c|c|c|c|c|c|c|c|}
\hline $\begin{array}{r}\text { Near IR excess } \\
(1.24-3.4 \mu \mathrm{m})\end{array}$ & $\begin{array}{r}\text { Mid IR excess } \\
(3.4-22 \mu \mathrm{m})\end{array}$ & $\begin{array}{r}\mathrm{H} \alpha \mathrm{EW} \\
(\AA)\end{array}$ & $\begin{array}{r}\mathrm{H} \alpha \\
\text { line shape }\end{array}$ & $V_{i}$ & UXOR & $\begin{array}{l}\text { Mass } \\
\left(M_{\odot}\right)\end{array}$ & $\begin{array}{r}\text { Age } \\
(\mathrm{Myr})\end{array}$ \\
\hline $0.46_{-0.19}^{+0.24}$ & $0.81_{-0.21}^{+0.27}$ & $-14.83 \pm 0.35^{33}$ & - & - & - & $1.90_{-0.26}^{+0.50}$ & $1.7_{-1.0}^{+1.6}$ \\
\hline $8.0_{-1.5}^{+2.2}$ & $12.6_{-3.0}^{-4.21}$ & $-95.3 \pm 4.4^{24}$ & - & - & - & $1.369_{-0.068}^{-0.068}$ & $17.2_{-3.6}^{+2.0}$ \\
\hline- & - & $-9.36 \pm 0.20^{15}$ & $\mathrm{~d}^{21}$ & - & - & - & - \\
\hline $0.077_{-0.037}^{+0.028}$ & $0.147_{-0.068}^{+0.049}$ & $-19.83 \pm 0.75^{27}$ & $d^{21}$ & - & - & - & - \\
\hline- & - & $-28.0 \pm 1.2^{15}$ & $d^{21}$ & - & - & - & - \\
\hline$\ldots$ & $\ldots$ & $\ldots$ & $\ldots$ & $\ldots$ & $\ldots$ & $\ldots$ & $\ldots$ \\
\hline
\end{tabular}

\begin{tabular}{rrrrrrrr}
\hline \hline$J$ excess & $H$ excess & $K_{\text {s excess }}$ & $W 1$ excess & $W 2$ excess & $W 3$ excess & W4 excess & The \\
$1.24 \mu \mathrm{m}$ & $1.66 \mu \mathrm{m}$ & $2.16 \mu \mathrm{m}$ & $3.4 \mu \mathrm{m}$ & $4.6 \mu \mathrm{m}$ & $12 \mu \mathrm{m}$ & - \\
\hline 1.49 & 2.28 & 4.20 & 10.16 & 22.35 & 168.78 & 2200.40 & Yes \\
14.73 & 49.13 & 170.17 & 325.14 & - & 7497.91 & 50750.83 & - \\
2.65 & 6.78 & 12.87 & - & - & - & 6594.26 & - \\
2.62 & 6.21 & 16.62 & 47.97 & 117.47 & 1245.27 & - & - \\
2.19 & 3.64 & 5.68 & - & - & $\ldots$ & $\ldots$ \\
$\ldots$ & $\ldots$ & $\ldots$ & $\ldots$ & $\ldots$ & $\ldots$ & $\ldots$ \\
\hline
\end{tabular}

Notes. This table is available in its entirety at the CDS. A portion is shown here for guidance regarding its form and content. Atmospheric parameters $T_{\text {eff }}, A_{\mathrm{V}}$ and $\mathrm{V}$ taken from the following sources in order of choice: Fairlamb et al. (2015), Montesinos et al. (2009), Hernández et al. (2004), Mendigutía et al. (2012), Carmona et al. (2010), Chen et al. (2016), Alecian et al. (2013), Sartori et al. (2010), Manoj et al. (2006), Hernández et al. (2005) and Vieira et al. (2003), APASS Data Release 9 and the SIMBAD database. If not available they were derived as described in Sect. 2.2. See Sect. 3 for derivation of $L$, Mass and Age. The $\mathrm{H} \alpha$ line profile classification is as follows: single-peaked (s), double-peaked (d) and showing a P-Cygni profile $(\mathrm{P})$, both regular or inverse. Variability indicator values $\left(V_{i}\right)$ for these objects could not be derived as they are not astrometrically well behaved. Similarly, many of these sources fall outside the Pre-Main Sequence tracks and isochrones in the HR diagram and no masses or ages could be derived for them. We decided to present the masses and ages in the cases they were computable but these values have to be taken with caution.

References. The references for binarity are: (1) Baines et al. (2006), (2) Wheelwright et al. (2010), (3) Leinert et al. (1997), (4) Maheswar et al. (2002), (5) Wheelwright et al. (2011), (6) Alecian et al. (2013), (7) Hamaguchi et al. (2008), (8) Dunhill et al. (2015), (9) Coulson \& Walther (1995), (10) Liu et al. (2000), (11) Biller et al. (2012), (12) Schütz et al. (2011), (13) Boersma et al. (2009), (14) Malkov et al. (2006), (15) Arellano Ferro \& Giridhar (2003), (16) Kubát et al. (2010), (17) Morrell \& Levato (1991), (18) Lazareff et al. (2017), (19) Mayer et al. (2016), (20) Folsom et al. (2008), (21) Corporon \& Lagrange (1999), (22) Doering \& Meixner (2009), (23) Chelli et al. (1995), (24) Miroshnichenko et al. (2002), (25) Friedemann et al. (1996), (26) Kraus et al. (2012), (27) Torres et al. (2000), (28) Aspin (1998), (29) Connelley et al. (2008), (30) Millour et al. (2009), (31) Frasca et al. (2016), (32) Marston \& McCollum (2008) and (33) Zhang et al. (2017). References for EW values and line shapes: (1) Fairlamb et al. (2017), (2) Carmona et al. (2010), (3) Mendigutía et al. (2011a), (4) Ababakr et al. (2016), (5) Hernández et al. (2004), (6) Baines et al. (2006), (7) Wheelwright et al. (2010), (8) van den Ancker et al. (1998), (9) Oudmaijer \& Drew (1999), (10) Kučerová et al. (2013), (11) Hernández et al. (2005), (12) Dunkin et al. (1997), (13) Pogodin et al. (2012), (14) Miroshnichenko et al. (1999), (15) Sartori et al. (2010), (16) Polster et al. (2012), (17) Manoj et al. (2006), (18) Miroshnichenko et al. (2004), (19) Miroshnichenko et al. (2002), (20) Borges Fernandes et al. (2007), (21) Vieira et al. (2003), (22) Boehm \& Catala (1995), (23) Nakano et al. (2012), (24) Spezzi et al. (2008), (25) Hou et al. (2016), (26) Grinin \& Rostopchina (1996), (27) Vieira et al. (2011), (28) Acke et al. (2005), (29) Herbig \& Bell (1988), (30) Oudmaijer et al. (1998), (31) X-Shooter spectra, 2015, priv. comm., from ESO observing program 084.C-0952A; (32) Ababakr et al. (2017), (33) Zuckerman et al. (2008), (34) Frasca et al. (2016), (35) Miroshnichenko et al. (1998) and (36) Miroshnichenko et al. (2000).

the points. The result is an estimate of age and mass for $236 / 252$ HAeBes. These values are presented in Tables 1 and 2 for the highand low-quality samples, respectively. Uncertainties were derived from the error bars in the HR diagram (Figs. 1 and 2) keeping a minimum error of 5\%. We compared many of our masses and ages with those of Alecian et al. (2013) and Reiter et al. (2018). We found that our determinations of these parameters are consistent with the results of the previous authors. 


\subsection{Infrared excesses}

In the process of deriving the luminosity it is also possible to derive the IR excess. We have logarithmically interpolated the different dereddened observed fluxes from the $J$ band $(1.24 \mu \mathrm{m})$ to the $W 4$ band $(22 \mu \mathrm{m})$ and defined the IR excess $(E)$ as:

$E=\frac{\left(F_{\mathrm{e}}-F_{*}\right)_{\left[\lambda_{1}, \lambda_{2}\right]}}{F_{*}}$,

where $F_{\mathrm{e}}$ is the total flux underneath the observed dereddened photometry (the IR photometry has also been dereddened) and $F_{*}$ is the total photospheric flux below the CK model. $\lambda_{1}$ and $\lambda_{2}$ define the range of wavelengths of interest and the total fluxes in the numerator simply refer to that range. This measure expresses the excess in terms of the total luminosity of the object. For example, all things being equal, if we have two stars with the same amount of dust surrounding them, with one of them brighter, the IR re-radiated emission will be larger but the IR excess, as defined here, would be the same, as it is a relative measure. The same or a very similar indicator was used by Cote \& Waters (1987, their Eq. (8)), Waters et al. (1987, Eq. (3)), and more recently by Banzatti et al. (2018) in their Sect. 2.3.

Uncertainties in the IR excesses were derived using the uncertainties in the observed fluxes and the uncertainties in the temperature (which affect the CK models) of each object.

We have split the total IR excess into two: a NIR excess (1.24-3.4 $\mu \mathrm{m}$, roughly the 2MASS region) and a MIR excess (3.4-22 $\mu \mathrm{m}$, the WISE region). The values for these excesses are presented in Tables 1 and 2 for the high- and low-quality samples, respectively. The total IR excess $(1.24-22 \mu \mathrm{m})$ is the sum of the two.

In addition, we also computed the IR excess at each individual band $\left(J, H, K_{\mathrm{s}}, W 1, W 2, W 3\right.$ and $\left.W 4\right)$ as the flux ratio between the dereddened observed monochromatic flux and the expected flux according to the $\mathrm{CK}$ model. The values for these excesses are presented in Tables 1 and 2 for the high- and low-quality samples, respectively

\subsection{Variability information}

Gaia DR2 does not provide a general variability indicator for all sources. Here, we use Gaia's repeated observations to extract photometric variability information. Gaia DR2 used a total of 22 months of observations and each source was observed repeatedly in a non-periodic fashion. Data Release 2 provides the average photometry, the uncertainty on this value, and the number of observations. All things being equal, the photometric "error" will be larger for a photometrically variable object than for a stable object. Here we aim to quantify the variability of the objects. We start with the "variability amplitude" $\left(A_{i}\right)$ for a certain source $i$ as presented in Deason et al. (2017):

$A_{i}=\sqrt{N_{\mathrm{obs}, i}} e\left(F_{i}\right) / F_{i}$,

where $N_{\mathrm{obs}}$ is the number of CCD crossings, and $F$ and $e(F)$ are the flux and flux error, respectively. This quantity is powerful in identifying objects that show larger flux variations than expected for a stable star. However, in order to statistically assess the level of variability, we introduce a variability indicator $V_{i}$, which quantifies how much more variable an object is compared to stable objects of the same brightness. In short it compares the variability amplitude from Eq. (3) of a given object ( $i$ ) to that of all Gaia objects in a brightness interval of \pm 0.1 magnitude around the $G$ band value of the object (i.e. to $A_{a, G_{a} \in\left(a_{1}, a_{2}\right)}$, with $a$ indexing the Gaia catalogue and being $a_{1}=G_{i}-0.1 \mathrm{mag}$ and $\left.a_{2}=G_{i}+0.1 \mathrm{mag}\right)$. The equation is as follows:

$V_{i}=\frac{A_{i}-\overline{A_{a}, G_{a} \in\left(a_{1}, a_{2}\right)}}{\sigma\left[A_{a}\right]_{G_{a} \in\left(a_{1}, a_{2}\right)}}$,

where $G$ is the Gaia white $G$ band magnitude and $\sigma$ is the standard deviation. In essence, we subtract the error to flux ratio of each $\mathrm{HAeBe}$, weighted by the number of observations, to the mean of the same expression $\left(A_{a}\right.$, Eq. (3)) for the sources in the Gaia catalogue within $\pm 0.1 \mathrm{mag}$ of the Herbig star in the $G$ band. We then divide by the standard deviation of $A_{a} s$ of that Gaia subset. This results in a variability indicator which measures the variability (in standard deviations, $\sigma$ ) for each Herbig $\mathrm{Ae} / \mathrm{Be}$ star compared to the mean of field objects of the same brightness.

For completeness, we note that it is necessary to impose more constraints to exclude the cases in which a larger error is not due to intrinsic variability. Following Deason et al. (2017), Appendix C of Lindegren et al. (2018) and what was done in Gaia Collaboration (2018a) we require $N_{\text {obs }} \geqslant 70$ and more than eight visibility periods (i.e. groups of observations separated by at least four days), in addition to the Eq. (1) constraint that limits the astrometric quality (and hence the variability indicator can simply be derived for sources in the highquality sample). In order to also limit the photometric quality, we included the following criterion presented in Gaia Collaboration (2018a):

$1.0+0.015\left(G_{\mathrm{BP}}-G_{\mathrm{RP}}\right)^{2}<E_{\mathrm{F}}<1.3+0.06\left(G_{\mathrm{BP}}-G_{\mathrm{RP}}\right)^{2}$,

where $E_{\mathrm{F}}$ is the flux excess factor and $G_{\mathrm{BP}}$ and $G_{\mathrm{RP}}$ the Gaia blue and red passbands, respectively. We note that these constraints may inevitably exclude many of the very variable HAeBes as they also trace larger errors and hence variability. These constraints will also be biased toward discarding binaries and faint sources in crowded areas (Lindegren et al. 2018; Gaia Collaboration 2018a).

The variability indicator values for the 193 sources satisfying the previous conditions are presented in Table 1.

In Fig. 3, we show the $V_{i}$ distribution of Herbig Ae/Be stars and compare it to the $V_{i}$ distribution of bright photometric standards from Landolt (2009) and faint photometric standards taken from Clem \& Landolt (2016). If Eq. (4) had not been used these two latter samples would have had a different mean in the distribution of $A_{i}$. The Herbig Ae/Be stars appear to show, on average, a larger variability indicator value than the standard stars, which have typical values of $V_{i} \lesssim 2$. We performed a two-sample Kolmogorov-Smirnov (KS) statistical test to decipher whether or not Herbig Ae/Be stars can be drawn from those two samples of standard stars. The result shows that we can reject that hypothesis to within a 0.001 significance and hence this variability indicator differentiates them as a group.

In order to assess the relation between our variability indicator ( $G$ band variability) and variability in the $V$ band we compared the magnitude variations in the $V$ band as presented in the International Variable Star Index VSX (Watson et al. 2006) with our variability indicator values. We found that we are tracing variabilities as small as $\sim 0.5 \mathrm{mag}$ with the $V_{i}=2$ cut-off. In Eiroa et al. (2002) 7/23 (30\%) PMS objects homogeneously observed for variability have variabilities above $0.5 \mathrm{mag}$. In our case 48 out of 193 sources have values above $V_{i}=2(25 \%)$ and 

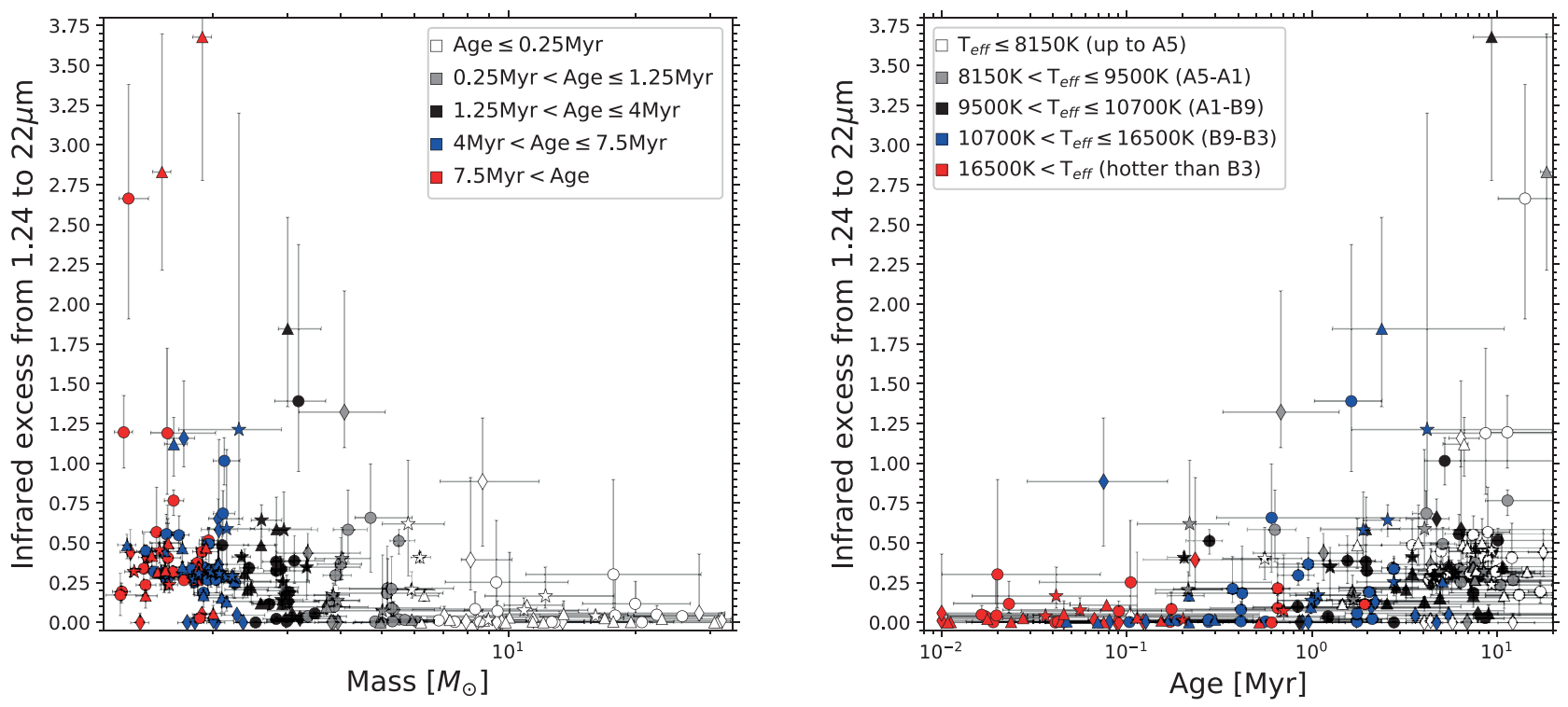

Fig. 4. Left panel: IR excess in the range 1.24-22 $\mu \mathrm{m}$ vs. estimated mass of the objects. The most massive objects (more massive than $\sim 7 M_{\odot}$ ) barely show an IR excess. Right panel: IR excess in the range 1.24-22 $\mu \mathrm{m}$ vs. estimated age. Ages and effective temperatures are respectively colour coded in the legend. The symbols stand for the $\mathrm{H} \alpha$ line profiles: circles (double-peaked), triangles (single-peaked), stars (P-Cygni profile) and diamonds (no information). We note that although it is not necessarily a one-to-one correlation, lower ages correspond to higher masses.

hence can be considered as strongly variable. Of those 48,17 are catalogued as UXOR type (Mendigutía 2011; Oudmaijer et al. 2001; Poxon 2015). There are 5 other UXORs in our sample with $V_{i}$ values, and 4 of them have reported optical variabilities smaller than $0.5 \mathrm{mag}$ in the $V$ band. The other one is BO Cep. This object has been reported to have a periodic variability with a single prominent peak with a period of $\sim 10$ days (Gürtler et al. 1999). The regular non-periodic variability of the object is smaller than 0.5 mag which explains why this UXOR has not been detected by our variability indicator. Supporting this, it appears as UXOR in Poxon (2015) but not in Oudmaijer et al. (2001) or Mendigutía (2011). Known UXORs are indicated in Tables 1 and 2 for the high- and low-quality samples, respectively.

To put the variability indicator into perspective, we find that 6 out of 411 photometric standards from Landolt (2009) have variability indicator values larger than 2 . We would therefore expect only 3 of our 193 Herbig Ae/Be stars for which we could determine $V_{i}$ to be strongly variable, at amplitudes of 0.5 magnitudes in the $V$ band or higher. However, we find 45 more, indicating that a large fraction of Herbig Ae/Be stars exhibit strong variations.

In addition, it is interesting to compare our variability indicator with the variability catalogues published alongside the Gaia DR2 general catalogue (Holl et al. 2018). Just 1 every 3000 objects passed the Gaia DR2 stringent selection criteria for variability. Ten of the 252 objects in our list fall in this category and appear as variable in those catalogues. Of the five of those that have derived $V_{i}$ values, they are larger than $V_{i}=5$.

\section{Data analysis}

\subsection{Infrared excesses}

In Fig. 4 the total IR excess (1.24-22 $\mu \mathrm{m})$ versus the estimated mass and age of the sources is plotted. There appears to be a difference in IR properties between high- and low-mass stars. Whereas low-mass stars show a range of IR excess, the highermass stars in general only present very low levels of excess. A similar behaviour is seen when the excess is plotted as a func-

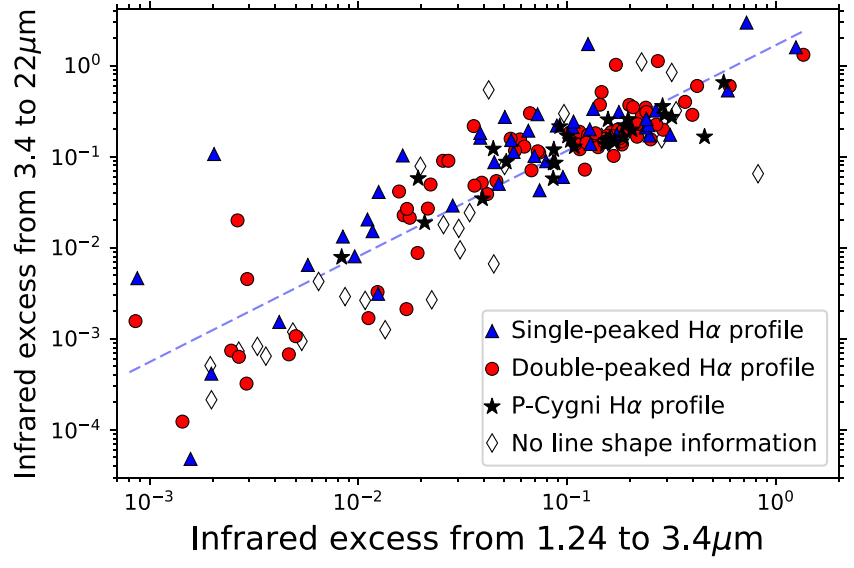

Fig. 5. IR excess in the range 3.4-22 $\mu \mathrm{m}$ (MIR excess) vs. IR excess in the range 1.24-3.4 $\mu \mathrm{m}$ (NIR excess). The symbols stand for the $\mathrm{H} \alpha$ line profiles: circles (double-peaked), triangles (single-peaked), stars (P-Cygni profile) and diamonds (no information). A linear fit in the log space is shown in blue $\left(\log \left(\operatorname{Mid} I R_{\text {excess }}\right)=1.16 \log \left(\right.\right.$ Near $\left.I R_{\text {excess }}\right)+0.23$, $r=0.88)$.

tion of age; the excess for the youngest objects is smallest. This is probably readily explained by the fact that the more massive PMS objects in the HR diagram have the lowest ages by virtue of their rapidly evolving isochrones, so trends in mass will automatically also be present in those with age. To study trends as a function of age, it would be necessary to consider subsamples with a narrow range in mass. We therefore consider that the main result of this exercise is that high-mass objects have a very low IR excess, and that there appears to be a break at $\sim 7 M_{\odot}$ from where almost no sources with significant excess appear.

Figure 5 splits the total IR excess into two, a NIR and a MIR part. This demonstrates that the excesses at both wavelength ranges are highly correlated with each other (the linear fit in logarithmic space that can be seen in the plot has a correlation coefficient of $r=0.88$ ). Therefore, it is not unexpected that the $\sim 7 M_{\odot}$ break is also present at NIR and MIR. 

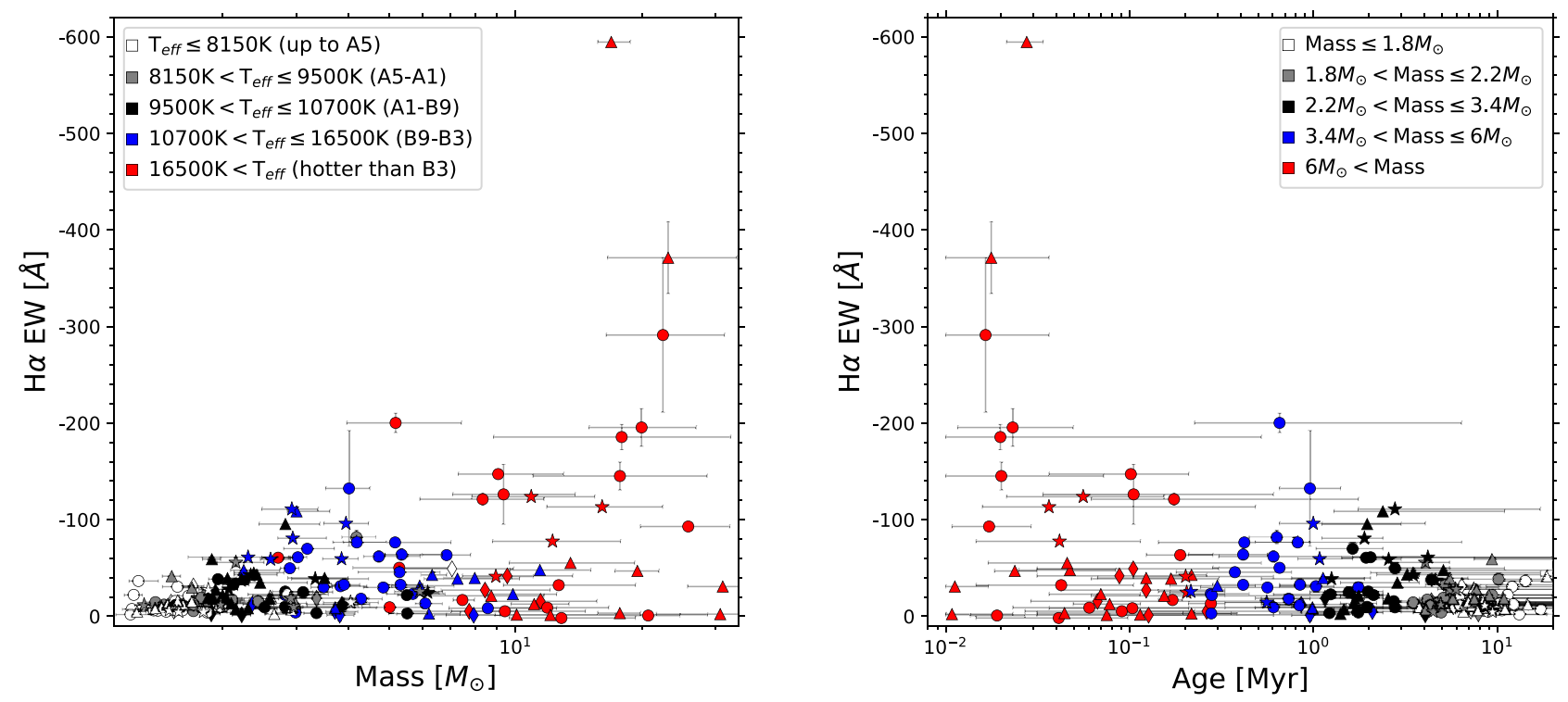

Fig. 6. Left panel: $\mathrm{H} \alpha \mathrm{EW}$ vs. estimated mass. Right panel: $\mathrm{H} \alpha \mathrm{EW}$ vs. estimated age. Effective temperatures and masses are respectively colour coded in the legend. The symbols stand for the $\mathrm{H} \alpha$ line profiles: circles (double-peaked), triangles (single-peaked), stars (P-Cygni profile) and diamonds (no information).

\section{2. $\mathrm{H} \alpha$ equivalent width}

Figure 6 shows the EW as a function of mass and age, respectively. As the definition of a Herbig Ae/Be star includes the presence of emission, which is mostly from the $\mathrm{H} \alpha$ line, it may not come as a surprise that essentially all measurements are negative (i.e. tracing emission).

The EWs show a large range of values, which appears to increase with increasing mass and decrease with increasing age (studied by Manoj et al. 2006). The older objects typically have lower EW's than younger objects. It is tempting to read an evolutionary effect into this finding - after all it would be expected that the accretion (and therefore emission) would decrease when the PMS objects are closer to the MS. However, we should recall that there is a strong correlation between the age and the mass of the stars, so we may well be looking at a mass effect instead. As the EW is a relative measurement with respect to the stellar continuum, a larger EW for otherwise similar objects indicates a stronger emission line. The observed trend towards higher temperatures/masses and thus higher luminosities implies that the lines become even stronger than the EW alone would seem to imply.

\subsection{Ho equivalent width and infrared excess}

The correlation between $\mathrm{H} \alpha$ emission, measured by its EW, and NIR and MIR excess is studied in Fig. 7 and Table 3 for each one of the IR bands $\left(J, H, K_{\mathrm{s}}, W 1, W 2, W 3\right.$ and W4). In this case, we computed the IR excess as the flux ratio between the dereddened observed monochromatic flux and the expected flux according to the CK model at each band (the values for these excesses are presented in Tables 1 and 2 for the high- and lowquality samples, respectively). In all cases, there is a general and consistent increase of the $\mathrm{H} \alpha \mathrm{EW}$ from sources with very little IR excess to those with higher IR excess.

In Table 3 we show that the $\mathrm{H} \alpha$ emission line EW is more correlated with the IR excess at shorter wavelengths than at larger wavelengths, with the correlation peaking at $2.16 \mu \mathrm{m}\left(K_{\mathrm{s}}\right.$ band $)$.

An obvious question might be whether there is a causal correlation between the $\mathrm{H} \alpha$ emission and presence of emission due to dust around these objects. The various excesses at various wavebands are correlated with each other (Fig. 5), and as a consequence the IR excesses at many wavelengths also correlate with the EW. However, the correlation with $\mathrm{H} \alpha$ is strongest at the $K_{\mathrm{s}}$ band which traces the hot dust in the inner disk, suggesting that the accretion mechanism or wind activity as traced by $\mathrm{H} \alpha$ is related to the inner parts of the dusty disk (see also Manoj et al. 2006). As presented in Table 3, the correlation rises from a minimum at $1.24 \mu \mathrm{m}$ (effectively tracing the stellar photosphere) up to $3.4 \mu \mathrm{m}$ and then goes down again to the same minimum at $22 \mu \mathrm{m}$ (W4 band), where dust in the outer disk is found. In fact, Mendigutía et al. (2012) discovered the same correlation between IR excess and accretion rate and they found that it is no longer present beyond $20 \mu \mathrm{m}$.

For comparison purposes, in Fig. 7 the $K_{\mathrm{s}}$ band is plotted in the upper panel and the $W 4$ band in the lower. It is noteworthy that for the $K_{\mathrm{s}}$ band, where we have the strongest correlation, small EWs are almost only present in sources with little IR excess and, in consonance with Sect. 4.1, for a given $\mathrm{H} \alpha$ EW value low-mass stars $\left(M<7 M_{\odot}\right)$ tend to have higher IR excesses. However, these trends are weaker or non-existent in the case of the W4 band, where we have the weaker correlation. This reinforces the idea that the $\mathrm{H} \alpha$ emission is correlated with the inner parts of the disk. We note that in both panels the average excess is still lower for the higher-mass objects. The emission line strengths will also be subject of a follow-up study using accretion rates (Wichittanakom et al. in prep.).

\subsection{Variability}

We conclude this section by studying the variability of the objects and its correlation with the various properties discussed so far, including the $\mathrm{H} \alpha$ line profiles taken from the literature.

The left panel of Fig. 8 presents the variability indicator as a function of the total (near plus mid) IR excess. As described in Sect. 3.4 the variability indicator states the number of standard deviations separating a certain source from the mean of the Gaia objects of the same brightness. No, or hardly any variability is 


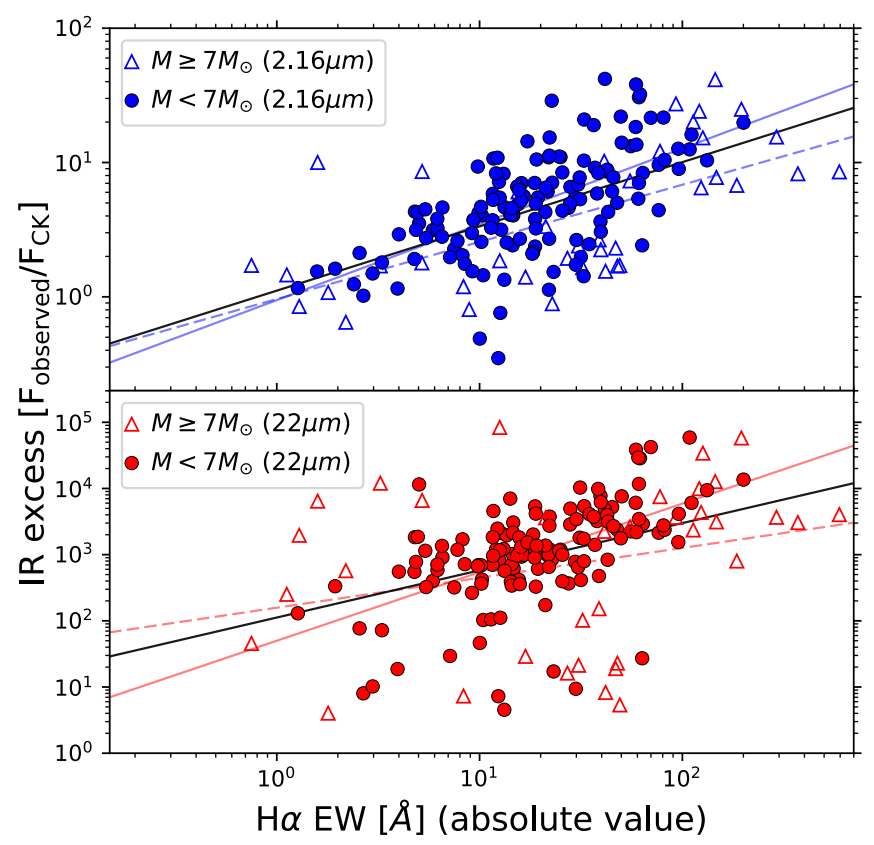

Fig. 7. $2.16 \mu \mathrm{m}$ (blue markers) and $22 \mu \mathrm{m}$ (red markers) IR excesses defined as $F_{\text {observed }} / F_{\text {CK }}$ vs. $\mathrm{H} \alpha$ EW (absolute value). We note that this IR excess indicator is a flux ratio and not the one described in Eq. (2) where we integrated under the SED. Dots are Herbig Ae/Be stars with $M<7 M_{\odot}$ and triangles are Herbig Ae/Be stars with $M>7 M_{\odot}$. Lines are linear fits to the data, dashed for HAeBes with $M>7 M_{\odot}$ and in solid colours for HAeBes with $M<7 M_{\odot}$; black solid lines are the linear fits for all the sources (equations and correlation coefficients for these fits to all the sources for all the IR bands can be seen in Table 3). We highlight the difference in the scale of the vertical axis between the two panels.

present at the lowest IR excesses but sources can be both variable and non-variable at the higher IR excesses, consistent with van den Ancker et al. (1998) based on a smaller sample.

The right panel of the same figure shows the variability as a function of mass. As high-mass stars in this sample generally do not have a strong IR excess, we find that mostly the lower-mass and cooler objects display high variabilities, with the break also around $7 M_{\odot}$, corresponding to a MS spectral type of around B3. Although cooler objects tend to have larger variabilities (also observed by van den Ancker et al. 1998), we can observe how the range in temperatures for variable sources is wide in the right panel of Fig. 8, and that there are in fact many Herbig Be stars with very strong variabilities. Therefore, this is more likely a trend with mass and not with temperature. We note that although we detect photometric variability from the $V_{i}=2$ value, the $V_{i}=$ 5 value is a better separation boundary for the observed trends in both panels of Fig. 8 .

The challenge is to identify which property lies at the cause of the variability; is it the mass of the objects, their age, or IR excess emission or something else? An important clue is that many objects with strong variability (above $V_{i}=2$ ) and line shape information have doubly peaked $\mathrm{H} \alpha$ profiles (31 out of $43 ; 72 \pm 7 \%, 68 \%$ confidence interval). In general, doublepeaked emission line profiles are due to rotating disks, so the data are suggestive of an edge-on disk-type orientation and structure (from the remaining 12 objects they all have a P-Cygni profile and none have a single-peaked profile). The number of variable objects with doubly peaked line profiles is significantly different from the full sample, in which only half of the targets with known line classifications have a double-peaked profile (of the
Table 3. Correlation between IR excess and $\mathrm{H} \alpha$ EW at different wavelengths.

\begin{tabular}{lrrr}
\hline \hline Band & $\begin{array}{r}\text { Correlation } \\
\text { coefficient }(r)\end{array}$ & $A$ & $B$ \\
\hline$J(1.24 \mu \mathrm{m})$ & 0.41 & $0.15 \pm 0.03$ & $0.025 \pm 0.034$ \\
$H(1.66 \mu \mathrm{m})$ & 0.56 & $0.32 \pm 0.03$ & $0.0024 \pm 0.0478$ \\
$K_{\mathrm{s}}(\mathbf{2 . 1 6} \mu \mathrm{m})$ & $\mathbf{0 . 6 0}$ & $\mathbf{0 . 4 8} \pm \mathbf{0 . 0 5}$ & $\mathbf{0 . 0 4 6} \pm \mathbf{0 . 0 6 6}$ \\
$W 1(3.4 \mu \mathrm{m})$ & 0.57 & $0.64 \pm 0.07$ & $0.15 \pm 0.10$ \\
$W 2(4.6 \mu \mathrm{m})$ & 0.57 & $0.78 \pm 0.09$ & $0.24 \pm 0.12$ \\
$W 3(12 \mu \mathrm{m})$ & 0.52 & $0.93 \pm 0.12$ & $0.79 \pm 0.16$ \\
$W 4(\mathbf{2 2} \mu \mathrm{m})$ & $\mathbf{0 . 4 1}$ & $\mathbf{0 . 7 1} \pm \mathbf{0 . 1 2}$ & $\mathbf{2 . 0 5} \pm \mathbf{0 . 1 7}$ \\
\hline
\end{tabular}

Notes. Correlation between IR excess (defined as a flux ratio, $\left.F_{\text {observed }} / F_{\mathrm{CK}}\right)$ and $\mathrm{H} \alpha \mathrm{EW}$ at different wavelengths for all the sources. The coefficients are defined by: $\log \left(\mathrm{F}_{\text {observed }} / \mathrm{F}_{\mathrm{CK}}\right)=A \log (|\mathrm{EW}|)+B$. The $K_{\mathrm{s}}$ band, with the higher correlation, and the $W 4$ band are in bold; both are shown in Fig. 7.

sources with derived variability indicator and known line profile, 79 out of 155 or $51 \pm 4 \%$ are double-peaked and 48 out of 155 or $31 \pm 4 \%$ are single-peaked). These fractions are significantly different, and we therefore suspect that the variable sources are mostly oriented edge-on, and that the line-of-sight inclination to the objects could be a decisive factor in the cause of the variability. This is in agreement with the trend observed in the left panel of Fig. 8. Sources with large amounts of circumstellar material show large IR excesses and high or low levels of variability depending on the inclination of their disk whilst sources with little material around have low IR excesses and low variabilities in all cases (also discussed in van den Ancker et al. 1998).

\section{Discussion}

\subsection{General findings}

In the above we have determined fundamental parameters such as temperature, mass, age, IR excess, variability and luminosity for a large sample of Herbig Ae/Be stars which was made possible due to the more than a factor of ten increase in available distances to these objects compared to Hipparcos. With the Gaia DR2 data, the majority of known Herbig Ae/Be stars could be placed in the HR diagram. We found the following:

- There are more low-mass objects than high-mass objects, with the high-mass objects mostly located close to the MS.

- High-mass objects have in general very small IR excesses and low variability; the properties appear to differ around $7 M_{\odot}$.

- $\mathrm{H} \alpha$ emission is generally correlated with IR excess, with the correlation stronger for IR emission at wavelengths tracing the hot dust closest to the star.

- More massive and younger objects have higher $\mathrm{H} \alpha$ EWs.

- When split at $7 M_{\odot}$ into "low" - and "high"-mass samples, the $\mathrm{H} \alpha$ - IR excess correlations hold for both mass ranges, with the average excess being lower for the higher-mass objects.

- Photometric variability can be traced back to those objects with double-peaked $\mathrm{H} \alpha$ emission and large IR excesses.

- All catalogued UXORs in the sample with detected variabilities above $0.5 \mathrm{mag}$ in the $V$ band appear as strongly variable (above $V_{i}=2$ ) with the exception of BO Cep (discussed in Sect. 3.4). 

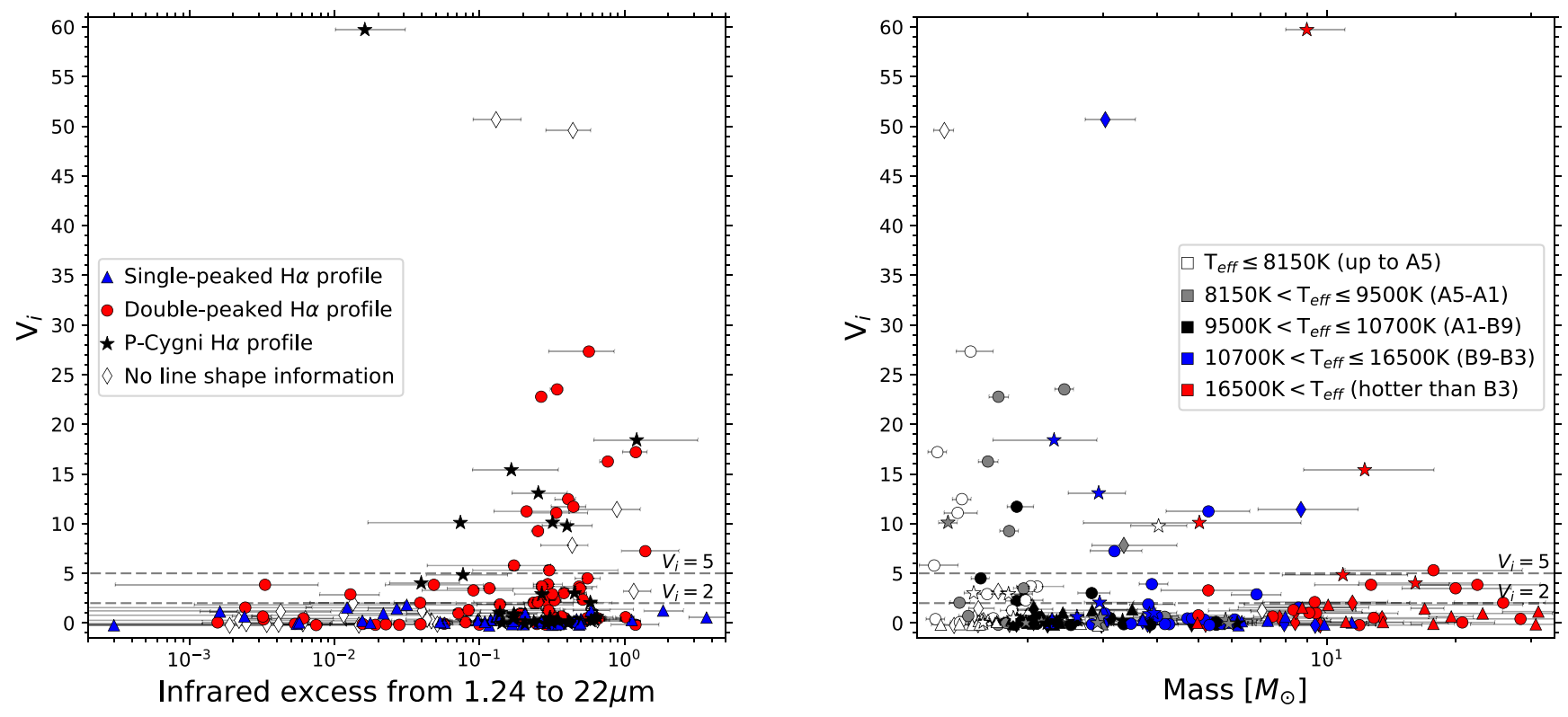

Fig. 8. Left panel: variability indicator vs. IR excess in the range 1.24-22 $\mu \mathrm{m}$. It can be seen how objects with the lower IR excess do not show high variability. Right panel: variability indicator vs. estimated mass. It can be seen how the most massive objects (more massive than $\sim 7 M_{\odot}$ ) barely show any variability. Line profiles and temperatures are colour coded in the legend in the left and right panels, respectively. The symbols stand for the $\mathrm{H} \alpha$ line profiles: circles (double-peaked), triangles (single-peaked), stars (P-Cygni profile) and diamonds (no information). The $V_{i}=2$ and $V_{i}=5$ values are stressed for clarity.

Below, we discuss these findings and their implications for the formation of intermediate-mass stars.

\subsection{Selection effects}

Let us first investigate the various selection effects and biases that could potentially affect the results.

Quality parallaxes. It could be argued that the quality of the astrometric data has an effect on the findings. The parallax errors occupy a comparatively small range, from $\sim 0.016-0.37$ mas, but because of the large spread in distances, the relative uncertainties can be very large. To investigate whether this has a detrimental effect on the results, we repeated the analysis with only the objects with the very best parallaxes $\left(\varpi / \sigma_{\varpi}>10\right)$. This, of course, limits the sample and 182 out of 218 objects remain in the high-quality sample. These 182 objects are less luminous, which may be expected as in general they have larger parallaxes and are therefore closer. As a result they will be less massive and have greater ages than the objects in the entire sample. This, as a consequence of the trends described in previous sections, implies that these objects also show larger IR excesses and variabilities as well as smaller $\mathrm{H} \alpha$ EWs (see Figs. 4, 6 and 8). However, we find that essentially all correlations also hold for the higherquality parallax sample, and if anything, they appear stronger. For example, almost all of the high-mass sources that have large IR excesses and variabilities in Figs. 4 and 8 have $\varpi / \sigma_{\varpi}<10$. The inclusion of lower-quality parallaxes induces an extra scatter in the results, but the larger sample and wider coverage in luminosity aids in reinforcing them.

Quality identification as Herbig Ae/Be star. Another potential source of error is source misclassification. We have used the largest sample of Herbig $\mathrm{Ae} / \mathrm{Be}$ stars published to date (Chen et al. 2016 with some added from Alecian et al. 2013; Baines et al. 2006; Carmona et al. 2010; Fairlamb et al. 2015;
Hernández et al. 2005; Manoj et al. 2006; Sartori et al. 2010). The defining characteristics of HAeBes are not unique to the class, and can often also be found in other types of stars such as classical Be stars, which display $\mathrm{H} \alpha$ emission and a NIR excess (e.g. Rivinius et al. 2013), and evolved stars, which can have spectral types $\mathrm{A}$ and $\mathrm{B}$, display hydrogen recombination emission, and be surrounded by dusty shells and disks such as the luminous blue variables and B[e] stars (Davies et al. 2007; Oudmaijer et al. 1998 on HD 87643). It is therefore inevitable that some sources will have been misclassified. It would be fair to say that the more "classical" Herbig Ae/Be stars going back to the Herbig (1960) and Thé et al. (1994) papers have been studied in more detail and are better established as young PMS stars.

We therefore studied the The et al. sample of objects (their Table 1, 85 sources out of our 218) separately and find that all correlations do hold for this "gold standard" sample as well. We do find that on average these objects have a larger $\mathrm{H} \alpha \mathrm{EW}$ and have larger IR excesses than the full sample. These properties are the defining characteristics of a Herbig Ae/Be star, and it may not be surprising that the first objects to be proposed as Herbig $\mathrm{Ae} / \mathrm{Be}$ stars are on average more extreme in these properties. Yet, again, as with the higher-quality parallax sample, the trends are still present in this sub-sample.

Mass distribution of the sample. The known Herbig Ae/Be stars have mostly been found serendipitously, and a large-scale systematic search for them has yet to be carried out. Yet, an interesting question is how representative the present sample is for the class. To this end, we consider the mass distribution of the objects. There are more or less the same number of low-mass, A-type objects as there are higher-mass B-type objects. There are more Herbig Be stars than might be expected from the IMF; however, the B-type objects are brighter and are sampled from a larger volume, as also attested by their smaller parallaxes. We would therefore expect a larger fraction of Herbig Be stars in the sample. When limiting our sample in distance, we obtain a Herbig Ae/Herbig Be ratio that is close to the IMF. As far as the 
mass distribution is concerned, we may say that the current sample is representative of the class. One of our future goals is to draw an increased and well-selected sampled of Herbig Ae/Be stars from the Gaia catalogues.

Binarity. One may think that binarity may affect the observed photometry and for example produce fake levels of variability in our variability indicator. This is because binary sources tend to be more astrometrically and photometrically irregular. We studied the group of binaries against the group of isolated sources and overall we find that the known binaries are slightly brighter than the objects that have not been reported to be a binary. This is probably a selection effect in that brighter objects were more likely to be included in the binary surveys. We compared the brightnesses of binaries and non-binaries in the Baines et al. (2006), Wheelwright et al. (2010) and Leinert et al. (1997) studies separately and find that within the surveys there are indeed no brightness differences between binaries and non-binaries.

Returning to the Gaia sample; all other properties but IR excesses, including variability, are similar. We do find that binaries have in general slightly larger IR excesses. With the benefit of hindsight, this is perhaps something that could have been expected. Most of the binaries are distant binaries with separations larger than 0.1 arcsec (Gaia's resolution). Indeed, no binaries are found closer than $30 \mathrm{au}$ and therefore binarity is not expected to play a significant role in the optical photometry. At the same time, companions could potentially contribute to the IR emission whose fluxes have been measured with apertures larger than the typical separations. Given that we do detect slight differences in IR excess between binaries and non-binaries, a preliminary inference would be that the companions may contribute to the IR flux in some cases.

\subsection{Infrared excess as a function of mass}

Figure 4 shows the IR excess as a function of mass and of age. There is a marked difference in the IR excess observed towards high- and low-mass objects. Herbig Be stars more massive than $\sim 7 M_{\odot}$ in general appear to have little to no excess, while the lower-mass objects show a wide range of excesses. There is also a trend with age with the youngest objects having the smallest IR excess. Although it would be tempting to assume a causal relation between age and presence of dust, and try to explain why the youngest objects have the smallest amount of dust around them, we suspect the stellar mass is the dominant factor. The durations of the PMS evolutionary tracks are progressively shorter for higher masses, and an underlying relation between mass and IR excess would therefore also appear as a correlation between age and IR excess.

Either way, the lack of dusty emission from high-mass objects is puzzling, as we might expect the more massive objects to be formed in more massive clouds and therefore be more embedded. A natural conclusion would be that at any time of their PMS evolution, these young objects would be surrounded by more dust than their lower-mass counterparts, and therefore, at any stage, they would have a stronger IR emission. A counterargument is that the Herbig Be stars are predominately found closer to the ZAMS and are therefore more evolved, having dispersed their circumstellar material. Supporting this idea, Alonso-Albi et al. (2009) found, from their compilation of millimetre observations of 44 objects, that Herbig Be stars have much weaker millimetre emission than their later-type counterparts. In addition, they found that the masses of the disks around Herbig Be stars traced at millimetre wavelengths are usually five to ten times lower than those around lower-mass stars, with the boundary also around $7 M_{\odot}$. These authors suggest that the disk dispersal is more efficient and faster in high-mass objects above $7 M_{\odot}$. Indeed, the disk dispersal times are a steep, declining function with stellar mass, from millions of years for the lower-mass stars to tens of thousands of years for the highest-mass young stars of $10 M_{\odot}$ and higher (Gorti et al. 2009).

The latter timescales are comparable to the evolutionary timescales as for example computed by Bressan et al. (2012) for these massive objects. Therefore, the observation here is consistent with the classical scenario that the Kelvin-Helmholtz contraction timescale is much smaller for massive objects compared to the free-fall timescale of the collapsing parental cloud. In this scenario, the massive young stars only become visible once they are on, or close to, the MS - the so-called birthline. We discuss this further below, but note that with this interpretation one would still expect a range of IR excesses in any sample. This is consistent with what we find for massive objects (larger than $7 M_{\odot}$ ); a large number of low-excess stars, but still a few with noticeable excess (see Fig. 4).

Moving to the lower-mass objects, which do display a large range of IR excess emission, an immediate question to ask is whether we can detect any evolutionary effect in the sense that objects that are further evolved have smaller IR excesses, as one expected from the progressive dust dispersal, and as suggested by Fuente et al. (1998). For example, if the inside-out clearing model of disk evolution is correct, we should see a trend at each PMS track from high excess to little excess.

However, it appears that Herbig Ae/Be stars do not show any consistent evolution of the IR excess from high to low excess at any mass range. There are many objects appearing younger than 2.5 Myr or even $1 \mathrm{Myr}$ at all mass ranges with little IR excess. Arguably the lack of an evolutionary effect can be explained by the size of the error bars on for example the luminosity. The evolutionary timescales vary strongly with mass (and thus luminosity), masking any trend of IR excess emission with age. Here, we would highlight that many young Herbig Ae stars show little excess. By looking at these objects in the right panel of Fig. 2 it is not difficult to find sources with error bars small enough to discard the contribution of uncertainty to the problem. Finally, the contamination by binaries as discussed in Sect. 5.2 can play a role here as many HAeBes can still remain as undetected binaries.

We should also note that the underlying assumption of the evolutionary calculations is that the conditions under which the stars form are uniform, and the accretion rates are a smooth function of time, resulting in an overall similar evolution for all stars. However, the final configuration is undoubtedly affected by inhomogeneities, varying accretion rates, and even the masses of the initial clouds. Nevertheless, looking for real evolutionary effects in the SEDs requires selecting subsamples of objects that are located at or close to the same mass tracks. In many cases this may require even more precise parallaxes than can presently be provided by Gaia. It also requires precise determinations of the atmospheric parameters and extinction values. A proper statistical study with high-quality parameters of the evolutionary properties of the HAeBes as they move towards the MS is therefore pending but is planned for the future.

\subsection{Variability in terms of the UXOR phenomenon}

The variability indicator that was developed specifically for the Gaia data demonstrates that the class of Herbig Ae/Be stars is more variable than the general population of stars. Figure 8 
shows that the lower-mass objects are much more photometrically variable than those of higher mass, for which the variability appears to cease beyond $\sim 7 M_{\odot}$. The photometrically variable objects contain most of the so-called UXOR variables reported in the literature. Using the compilation of UXOR variables by Mendigutía (2011), Oudmaijer et al. (2001) and Poxon (2015), we find that 17 out of the 48 strongly variable objects those with variability indicator values larger than 2 , representing variations of 0.5 magnitudes (in the $V$ band) or higher - are classified as UXORs. The remaining 5 UXORs with variability indicator values present in the sample have documented variabilities below 0.5 magnitudes with the exception of BO Cep (discussed in Sect. 3.4).

The defining characteristic of the UXOR phenomenon is not only the photometric variability but also the reddening and blueing associated during the variations. The explanation put forward for this behaviour is the obscuration of the star by a rotating, inhomogeneous, dusty, edge-on disk. The objects first become redder when dust obscures the object, and can even become blue at their faintest phases, when the direct light from the stars is blocked and, predominately blue light is scattered into the line of sight. As the polarization - resulting from scattered light - also peaks during the faintest phases (e.g. Grinin 2000), the obscuring disk hypothesis is favoured. Interestingly, observational evidence other than the polarization supporting this conclusion has been relatively sparse.

With the large sample of Herbig Ae/Be stars, and the large number of UXORs among them, we can repeat a similar experiment using the $\mathrm{H} \alpha$ line as a proxy for the inclination of the circumstellar disks. We consider the line profiles of the $\mathrm{H} \alpha$ emission in tandem with the variability indicator. Figure 8 shows that all but twelve of the strongly variable objects with documented line profiles (above $V_{i}=2$, those with $\Delta V>0.5 \mathrm{mag}$ ) have double-peaked $\mathrm{H} \alpha$ emission. In fact, the five objects for which no line profile is listed have, to our knowledge, no reported profiles. The occurrence of double-peaked profiles in the highly variable sample is significantly higher than for the other objects (see Sec. 4.4). It is significant that the other twelve objects have P-Cygni profiles and none of them show a single-peaked profile. The P-Cygni profile is often related to episodic energetic phenomena and it is not unexpected that it is also traced by our variability indicator. Given that doubly peaked line profiles are most easily explained by at least part of the emission originating in a rotating disk leads us to conclude that the photometrically variable objects are seen edge-on and surrounded by a disk-like structure. It is true that outflows or winds not limited to the disk can produce double-peaked $\mathrm{H} \alpha$ profiles (Kurosawa et al. 2006; Tambovtseva et al. 2014). Supporting the hypothesis of edge-on disks being the main cause of photometric variability, we find in variability the same separation at $\sim 7 M_{\odot}$ between low- and high-mass objects that we found when studying IR excesses, which suggests that photometric variability and IR excess have the same cause. In addition, sources with high IR excesses have both high and low variability levels, which can be understood as depending on the disk inclination, while sources with lower IR excesses show little variability in all cases (left panel of Fig. 8, discussed in Sect. 4.4). This would also explain the few highmass strongly variable objects that can be seen in the right panel of Fig. 8; they are mostly the ones with high IR excess in the left panel of Fig. 4 (discussed before in Sect. 5.3). Given that an edge-on orientation is the major and main ingredient of the dust obscuration hypothesis, these results lend very strong support to it using a large sample of Herbig Ae/Be stars.
The large fraction of objects with double-peaked line profiles or variability is in agreement with the model predictions by Natta \& Whitney (2000) who worked out how many Herbig $\mathrm{Ae} / \mathrm{Be}$ stars would undergo the UXOR phenomenon considering the scale heights of dusty disks and under which inclinations the photometric variability would still be visible. They conclude that around half of the Herbig Ae stars could be UXORs. In our high-quality sample we have 85 A-type stars with variability indicator values and just 16 of them were previously listed as UXORs; however, again, most of them have been largely unstudied. Nevertheless, of the 25 A-type stars with variabilities above $V_{i}=2,13$ are known UXORs. This means that for the Herbig Ae stars for which we detect variability at the $V_{i}=2$ level, $\sim 52 \%$ are known UXORs (and just two have P-Cygni profiles). Moreover, this implies that we are retrieving $\sim 81 \%$ of known A-type UXORs with our variability indicator and hence, assuming that all the 25 A-type stars with variabilities above $V_{i}=2$ are of UXOR type; 31 of the 85 A-type stars with variability indicator values in the sample should be UXORs. In turn this would imply that $\sim 37 \%$ of all Herbig Ae stars belong to the UXOR class. If we also take into account that we have potentially removed some UXORs from consideration, possibly the most variable ones, when applying the constraints described in Sect. 3.4, we get to values close to the $50 \%$ predicted by Natta \& Whitney (2000).

Finally, Davies et al. (2018) recently studied the UXOR object $\mathrm{CO}$ Ori in detail, which has single-peaked $\mathrm{H} \alpha$ emission. Consequently, they found that the inclination of its disk is of $\sim 30^{\circ}$ (i.e. it is nearly face-on). In this particular case, whether the disk is still causing the UXOR phenomenon or if it is caused through fluctuations in the circumstellar material outside the disk is still uncertain. We could not derive a variability indicator value for this object to assess its variability. Inspired by this example, we took a look at the other UXORs in our sample with singlepeaked profiles; they all have variabilities below $V_{i}=2$ in our variability indicator (HD 100546, HD 142527, HD 98922 and IL Cep), suggesting a category of low-variability UXORs with nearly face-on disks. Nonetheless, the results presented in this section strongly support the idea that most UXORs are caused by edge-on disks, which are responsible for large photometric variabilities.

\subsection{Missing objects in the HR diagram}

When inspecting the right panel of Fig. 2, it appears that most Herbig Be stars are located relatively close to the MS, whereas the lower-mass Herbig Ae stars occupy a larger part of their evolutionary tracks, contracting to higher temperatures at constant luminosity. In other words, the late-type Herbig Be and Herbig Ae stars at high luminosities (and low surface gravities) that would occupy the tracks towards the locations of B-type stars on the MS are missing. It is only due to the use of Gaia parallaxes, expanding the number of Herbig Ae/Be stars with well established luminosities, that we can make this observation.

In our discussion above, we mentioned the fact that these objects could still be heavily embedded in their parental clouds, preventing them from being optically visible when evolving on their way to the MS. There is evidence for optically invisible but IR-bright objects at locations in these regions of the HRdiagram. For example, Pomohaci et al. (2017) were the first to spectrally type an IR-bright MYSO based on the rare absorption spectrum at NIR wavelengths (higher-order Brackett lines are in absorption for this object, while $\mathrm{Br} \gamma$ is in emission). They 
found that the object could be fitted with the spectra of an Atype giant star. Had this object been optically visible, it would have occupied the empty region in the right panel of Fig. 2. To this, we add the early B-type Herbig Be stars/IR bright MYSOs PDS 27 and PDS 37 (Ababakr et al. 2015). They are found in the upper regions of the HR diagram, slightly off the MS. They are optically visible, but not overly bright at $V \sim 13 \mathrm{mag}$, and have not been included in many (optical) magnitude-limited catalogues. Therefore, there are several examples that might lead us to conclude that the - implicit - optical brightness limit of any catalogue of Herbig Ae/Be stars would prevent the inclusion of massive PMS stars on the horizontal portions of the evolutionary PMS tracks. However, these object are present in Gaia DR2 although they are yet uncatalogued as HAeBes. In a sense this is a situation similar to that outlined for the low IR excesses observed toward the Herbig Be stars that are mostly located close to the MS. This could be explained by the fact that the objects would be embedded and thus optically invisible or faint in earlier phases of their evolution.

Further observations of optically fainter objects will be necessary to settle this issue. Additional progress can be made by connecting the PMS evolutionary tracks with radiative transfer codes to provide synthetic observations (as e.g. Davies et al. 2011, or Zhang et al. 2014 for Massive Young Stellar Objects) extended to optical wavelengths in the Herbig Be mass range. Related to the "missing" high-mass stars in the HR diagram, it will be important to fill the historic, and entirely man-made, gap between the Herbig Ae stars and the T-Tauri stars. The latter are confined to have spectral types G-K-M, and typically Herbig $\mathrm{Ae} / \mathrm{Be}$ stars, in this case by definition, have spectral types $\mathrm{A}$ and B. We are missing out the F-type stars, resulting in an incomplete coverage of the HR diagram for PMS stars.

\subsection{The difference between Herbig Ae and Herbig Be stars}

From the above it appears that the dusty disks surrounding Herbig Ae and Herbig Be stars are different, with the break in IR excess occurring at $7 M_{\odot}$ (around B3 spectral type), a value which was also found by Alonso-Albi et al. (2009) from their compilation of millimetre emission tracing the outer parts of the dusty disks. As discussed, given the much stronger radiation field from B-stars, both in intensity and photon-energies, the most straightforward explanation for the much less massive disks of higher-mass objects is a more efficient disk dispersal mechanism (see e.g. Gorti et al. 2009). This also explains why the same $7 M_{\odot}$ break is seen in variability (Fig. 8). As described in Sect. 5.4, the high levels of variability in some sources are caused by edge-on dusty disks. A more efficient disk dispersal mechanism beyond $7 M_{\odot}$ would result in these sources showing no strong variability in our indicator. It also explains why the objects with the lower IR excesses are not strongly variable while the rest can have both high and low variability values.

Other studies of large samples of Herbig Ae/Be stars indicate a break in properties at a much lower mass of $3 M_{\odot}$, around the B7 spectral boundary. Fairlamb et al. (2015) studied the accretion rates, which are proportional to the mass of the objects, and found a different slope for lower-mass than for higher-mass objects. Ababakr et al. (2017), extending the work of Mottram et al. (2007), found a distinct difference in spectropolarimetric properties across the $\mathrm{H} \alpha$ line between the Herbig Ae and late Be-type stars on the one hand and earlier Herbig Be-type objects on the other hand. These authors also point out the similarity in the $\mathrm{H} \alpha$ spectro-polarimetry of the Herbig Ae stars and T-Tauri stars. Finally, Mendigutía et al. (2011a) noted the difference in $\mathrm{H} \alpha$ variability; Herbig Ae and late Be stars are largely variable, whereas Herbig Be stars are not. Later, Fang et al. (2013) showed that T-Tauri stars display even more variable $\mathrm{H} \alpha$ emission - again hinting at a similar accretion mechanism for the T-Tauri stars and Herbig Ae stars.

How can we reconcile the fact that some studies show a break in properties that is different from that of others? It is worth pointing out that the latter investigations consider regions much closer to the star than the dusty emission. Fairlamb et al. (2015) derive accretion rates from the UV excess which trace the shocked material on the stellar surface, and Ababakr et al. (2017)'s spectropolarimetry traces the free electrons in ionized material at distances of the order of stellar radii from the stars. The spectro-polarimetric properties of the B-type stars can be explained by stable circumstellar disks, while the line properties for T-Tauri and Herbig Ae objects are consistent with magnetically controlled accretion. Likewise, the $\mathrm{H} \alpha$ emission traces the ionized zones close to the star, such as the accretion columns and circumstellar disks, and the variability is explained by the accretion columns orbiting the central star (e.g. Kurosawa et al. 2008).

Earlier, we showed that the IR fluxes and $\mathrm{H} \alpha$ properties are largely correlated, but that the IR fluxes are smaller for the earlier-type objects. We therefore conclude this section with the observation that the IR and millimetre emissions trace the circumstellar disks and originate much further from the stars than the UV, hydrogen recombination emission, and free electrons, which themselves trace the accretion onto the stars. The break in accretion mechanism appears to occur around $3 M_{\odot}$, whereas the disk dispersal becomes significant at higher masses of $7 M_{\odot}$.

\section{Conclusions}

In this paper we have collated the largest astrometric dataset of Herbig Ae/Be stars. We present parallaxes for the vast majority of known Herbig Ae/Be stars and have gathered atmospheric parameters, optical and IR photometry, extinction values, $\mathrm{H} \alpha$ emission line information, and binary statistics, and have devised an objective measure for the photometric variability. From these we derive luminosities which allow us to place the objects in a HR diagram, containing over ten times more objects than previously possible.

Thus, we homogeneously derived luminosities, distances, masses, ages, variabilities and IR excesses for the most complete sample of Herbig Ae/Be stars to date. We investigated the various properties and reach the following conclusions.

1. The Gaia photometric variability indicator as developed here indicates that 48 out of 193 or $\sim 25 \%$ of all Herbig Ae/Be stars are strongly variable. We find that the presence of variability correlates very well with the $\mathrm{H} \alpha$ line profile. The variable objects display doubly peaked profiles, indicating an edge-on disk. It had been suggested that this variability is in most cases due to asymmetric dusty disk structures seen edge-on. The observation here is the most compelling confirmation of this hypothesis. Most sources catalogued as UXORs in the sample appear as strongly variable with double-peaked profiles. The fraction of strongly variable Atype objects is close to that found for the A-type objects with the UXOR phenomenon.

2. High-mass stars do not display an IR excess and show no strong photometric variability. Several suggestions have been put forward to explain this. These include fast evolutionary timescales and fast dust dispersion timescales for high-mass objects. We note that the break is around $7 M_{\odot}$, 
which is intriguingly similar to other statistical studies related to dusty disks around Herbig Ae/Be stars which signpost a different or more efficient disk dispersal mechanism for high-mass objects.

3. Whereas the break in IR properties and photometric variabilities occurs at $7 M_{\odot}$, various $\mathrm{H} \alpha$ line properties including mass accretion rates, spectropolarimetric properties, and emission line variability seem to differ at a lower mass of $3 M_{\odot}$. The latter has been linked to different accretion mechanisms at work; magnetospheric accretion for the Atype objects and another mechanism, possibly boundary layer accretion, for the B-type objects. The differing IR and variability properties are related to different or differently acting (dust-)disk-dispersal mechanisms, which occurs at much larger size scales than the accretion traced by hydrogen recombination line emission.

Finally, the findings presented in this paper signal just the beginning in unveiling the formation of intermediate-mass stars using Gaia. Gaia presents us with an excellent opportunity to search and identify new Herbig Ae/Be stars, resulting in a well-selected and properly characterized sample. The results presented here will assist greatly in identifying new Herbig Ae/Be objects from the more than one billion stars with astrometric parameters in Gaia. This is the subject of our follow-on study, the STARRY project.

Acknowledgements. The STARRY project has received funding from the European Union's Horizon 2020 research and innovation programme under MSCA ITN-EID grant agreement No 676036. This work has made use of data from the European Space Agency (ESA) mission Gaia (https://www. cosmos.esa.int/gaia), processed by the Gaia Data Processing and Anal ysis Consortium (DPAC, https://www. cosmos.esa.int/web/gaia/dpac/ consortium). Funding for the DPAC has been provided by national institutions, in particular the institutions participating in the Gaia Multilateral Agreement This research also made use of Astropy, a community-developed core Python package for Astronomy (Astropy Collaboration 2013), the TOPCAT tool (Taylo 2005) and the VizieR catalogue access tool and the SIMBAD database, operated at CDS, Strasbourg, France. This research was made possible through the use of the AAVSO Photometric All-Sky Survey (APASS), funded by the Robert Martin Ayers Sciences Fund. We thank the referee for his/her insightful comments which have improved the paper.

\section{References}

Aarnio, A. N., Monnier, J. D., Harries, T. J., et al. 2017, ApJ, 848, 18 Ababakr, K. M., Fairlamb, J. R., Oudmaijer, R. D., \& van den Ancker, M. E. 2015, MNRAS, 452, 2566

Ababakr, K. M., Oudmaijer, R. D., \& Vink, J. S. 2016, MNRAS, 461, 3089 Ababakr, K. M., Oudmaijer, R. D., \& Vink, J. S. 2017, MNRAS, 472, 854 Acke, B., van den Ancker, M. E., \& Dullemond, C. P. 2005, A\&A, 436, 209 Alecian, E., Wade, G. A., Catala, C., et al. 2013, MNRAS, 429, 1001 Alonso-Albi, T., Fuente, A., Bachiller, R., et al. 2009, A\&A, 497, 117 Arellano Ferro, A., \& Giridhar, S. 2003, A\&A, 408, L29

Arenou, F., Luri, X., Babusiaux, C., et al. 2018, A\&A, 616, A17

Aspin, C. 1998, A\&A, 335, 1040

Astropy Collaboration (Robitaille, T. P., et al.) 2013, A\&A, 558, A33

Bailer-Jones, C. A. L. 2015, PASP, 127, 994

Bailer-Jones, C. A. L., Rybizki, J., Fouesneau, M., et al. 2018, ApJ, submitted

Baines, D., Oudmaijer, R. D., Porter, J. M., \& Pozzo, M. 2006, MNRAS, 367, 737

Banzatti, A., Garufi, A., Kama, M., et al. 2018, A\&A, 609, L2

Biller, B., Lacour, S., Juhász, A., et al. 2012, ApJ, 753, L38

Boehm, T., \& Catala, C. 1995, A\&A, 301, 155

Boersma, C., Peeters, E., Martín-Hernández, N. L., et al. 2009, A\&A, 502, 175

Borges Fernandes, M., Kraus, M., Lorenz Martins, S., \& de Araújo, F. X. 2007 MNRAS, 377, 1343

Bouvier, J., Alencar, S. H. P., Harries, T. J., Johns-Krull, C. M., \& Romanova, M. M. 2007, Protostars and Planets V, 479

Bressan, A., Marigo, P., Girardi, L., et al. 2012, MNRAS, 427, 127

Cardelli, J. A., Clayton, G. C., \& Mathis, J. S. 1989, ApJ, 345, 245

Carmona, A., van den Ancker, M. E., Audard, M., et al. 2010, A\&A, 517, A67
Castelli, F., \& Kurucz, R. L. 2004, ArXiv e-prints [arXiv: astro-ph/0405087] Cauley, P. W., \& Johns-Krull, C. M. 2015, ApJ, 810, 5

Chelli, A., Cruz-Gonzalez, I., \& Reipurth, B. 1995, A\&AS, 114, 135

Chen, P. S., Shan, H. G., \& Zhang, P. 2016, New Astron., 44, 1

Clem, J. L., \& Landolt, A. U. 2016, AJ, 152, 91

Connelley, M. S., Reipurth, B., \& Tokunaga, A. T. 2008, AJ, 135, 2496

Corporon, P., \& Lagrange, A.-M. 1999, A\&AS, 136, 429

Costigan, G., Vink, J. S., Scholz, A., Ray, T., \& Testi, L. 2014, MNRAS, 440 3444

Coulson, I. M., \& Walther, D. M. 1995, MNRAS, 274, 977

Coté, J., \& Waters, L. B. F. M. 1987, A\&A, 176, 93

Cutri, R. M., Wright, E. L., Conrow, T., et al. 2013, Explanatory Supplement to the AllWISE Data Release Products

Davies, B., Oudmaijer, R. D., \& Sahu, K. C. 2007, ApJ, 671, 2059

Davies, B., Hoare, M. G., Lumsden, S. L., et al. 2011, MNRAS, 416, 972

Davies, C. L., Kreplin, A., Kluska, J., et al. 2018, MNRAS, 474, 5406

Deason, A. J., Belokurov, V., Erkal, D., Koposov, S. E., \& Mackey, D. 2017, MNRAS, 467, 2636

Doering, R. L., \& Meixner, M. 2009, AJ, 138, 780

Duchêne, G. 2015, Ap\&SS, 355, 291

Dullemond, C. P., \& Dominik, C. 2004a, A\&A, 417, 159

Dullemond, C. P., \& Dominik, C. 2004b, A\&A, 421, 1075

Dullemond, C. P., \& Dominik, C. 2005, A\&A, 434, 971

Dunhill, A. C., Cuadra, J., \& Dougados, C. 2015, MNRAS, 448, 3545

Dunkin, S. K., Barlow, M. J., \& Ryan, S. G. 1997, MNRAS, 290, 165

Eiroa, C., Oudmaijer, R. D., Davies, J. K., et al. 2002, A\&A, 384, 1038

Evans, D. W., Riello, M., De Angeli, F., et al. 2018, A\&A, 616, A4

Fairlamb, J. R., Oudmaijer, R. D., Mendigutía, I., Ilee, J. D., \& van den Ancker, M. E. 2015, MNRAS, 453, 976

Fairlamb, J. R., Oudmaijer, R. D., Mendigutía, I., Ilee, J. D., \& van den Ancker, M. E. 2017, MNRAS, 464, 4721

Fang, M., Kim, J. S., van Boekel, R., et al. 2013, ApJS, 207, 5

Finkenzeller, U., \& Mundt, R. 1984, A\&AS, 55, 109

Folsom, C. P., Wade, G. A., Kochukhov, O., et al. 2008, Contributions of the Astronomical Observatory Skalnate Pleso, 38, 245

Frasca, A., Miroshnichenko, A. S., Rossi, C., et al. 2016, A\&A, 585, A60

Friedemann, C., Guertler, J., \& Loewe, M. 1996, A\&AS, 117, 205

Fuente, A., Martin-Pintado, J., Bachiller, R., Neri, R., \& Palla, F. 1998, A\&A, 334,253

Gaia Collaboration (Prusti, T., et al.) 2016, A\&A, 595, A

Gaia Collaboration (Babusiaux, C., et al.) 2018a, A\&A, 616, A10

Gaia Collaboration (Brown, A. G. A., et al.) 2018b, A\&A, 616, A1

Gorti, U., Dullemond, C. P., \& Hollenbach, D. 2009, ApJ, 705, 1237

Grady, C. A., Hamaguchi, K., Schneider, G., et al. 2010, ApJ, 719, 1565

Gray, R. O., \& Corbally, C. J. 2009, Stellar Spectral Classification, eds. Richard

O. Gray, \& Christopher J. Corbally (Princeton University Press)

Grinin, V. P. 2000, Disks, Planetesimals, and Planets, 219, 216

Grinin, V. P., \& Rostopchina, A. N. 1996, Astron. Rep., 40, 17

Grundstrom, E. D., \& Gies, D. R. 2006, ApJ, 651, L53

Gürtler, J., Friedemann, C., Reimann, H.-G., et al. 1999, A\&AS, 140, 293

Hamaguchi, K., Choi, M., Corcoran, M. F., et al. 2008, ApJ, 687, 425

Herbig, G. H. 1960, ApJS, 4, 337

Herbig, G. H., \& Bell, K. R. 1988, Third Catalog of Emission-line Stars of the Orion Population (Santa Cruz: Lick Observatory), Lick Observatory Bulletin, 1111

Hernández, J., Calvet, N., Briceño, C., Hartmann, L., \& Berlind, P. 2004, AJ, 127,1682

Hernández, J., Calvet, N., Hartmann, L., et al. 2005, AJ, 129, 856

Holl, B., Audard, M., Nienartowicz, K., et al. 2018, A\&A, 618, A30

Honda, M., Maaskant, K., Okamoto, Y. K., et al. 2015, ApJ, 804, 143

Hou, W., Luo, A.-L., Hu, J.-Y., et al. 2016, Res. Astron. Astrophys., 16, 138

Ilee, J. D., Fairlamb, J., Oudmaijer, R. D., et al. 2014, MNRAS, 445, 3723

Joner, M. D., \& Hintz, E. G. 2015, AJ, 150, 204

Klement, R., Carciofi, A. C., Rivinius, T., et al. 2017, A\&A, 601, A74

Kraus, S. 2015, Ap\&SS, 357, 97

Kraus, S., Calvet, N., Hartmann, L., et al. 2012, ApJ, 746, L2

Kubát, J., Saad, S. M., Kawka, A., et al. 2010, A\&A, 520, A103

Kučerová, B., Korčáková, D., Polster, J., et al. 2013, A\&A, 554, A143

Kurosawa, R., Harries, T. J., \& Symington, N. H. 2006, MNRAS, 370, 580

Kurosawa, R., Romanova, M. M., \& Harries, T. J. 2008, MNRAS, 385, 1931

Landolt, A. U. 2009, AJ, 137, 4186

Lazareff, B., Berger, J.-P., Kluska, J., et al. 2017, A\&A, 599, A85

Lee, C.-D., Chen, W.-P., \& Liu, S.-Y. 2016, A\&A, 592, A130

Leinert, C., Richichi, A., \& Haas, M. 1997, A\&A, 318, 472

Lindegren, L., Hernández, J., Bombrun, A., et al. 2018, A\&A, 616, A2

Liu, Q. Z., van Paradijs, J., \& van den Heuvel, E. P. J. 2000, A\&AS, 147, 25

Lumsden, S. L., Hoare, M. G., Urquhart, J. S., et al. 2013, ApJS, 208, 11

Luri, X., Brown, A. G. A., \& Sarro, L. M., et al. 2018, A\&A, 616, A9 
Maaskant, K. M., Honda, M., Waters, L. B. F. M., et al. 2013, A\&A, 555, A64 Maheswar, G., Manoj, P., \& Bhatt, H. C. 2002, A\&A, 387, 1003

Malkov, O. Y., Oblak, E., Snegireva, E. A., \& Torra, J. 2006, A\&A, 446, 785

Manoj, P., Bhatt, H. C., Maheswar, G., \& Muneer, S. 2006, ApJ, 653, 657

Marconi, M., \& Palla, F. 1998, ApJ, 507, L141

Marigo, P., Girardi, L., Bressan, A., et al. 2017, ApJ, 835, 77

Marston, A. P., \& McCollum, B. 2008, A\&A, 477, 193

Mayer, A., Deschamps, R., \& Jorissen, A. 2016, A\&A, 587, A30

Meeus, G., Waters, L. B. F. M., Bouwman, J., et al. 2001, A\&A, 365, 476

Mendigutía, I. 2011, PhD Thesis, Universidad Autónoma de Madrid, Spain

Mendigutía, I., Eiroa, C., Montesinos, B., et al. 2011a, A\&A, 529, A34

Mendigutía, I., Calvet, N., Montesinos, B., et al. 2011b, A\&A, 535, A99

Mendigutía, I., Mora, A., Montesinos, B., et al. 2012, A\&A, 543, A59

Millour, F., Chesneau, O., Borges Fernandes, M., et al. 2009, A\&A, 507, 317

Miroshnichenko, A. S., Fremat, Y., Houziaux, L., et al. 1998, A\&AS, 131, 469

Miroshnichenko, A. S., Gray, R. O., Vieira, S. L. A., Kuratov, K. S., \& Bergner, Y. K. 1999, A\&A, 347, 137

Miroshnichenko, A. S., Chentsov, E. L., Klochkova, V. G., et al. 2000, A\&AS, 147,5

Miroshnichenko, A. S., Bjorkman, K. S., Chentsov, E. L., et al. 2002, A\&A, 388, 563

Miroshnichenko, A. S., Levato, H., Bjorkman, K. S., et al. 2004, A\&A, 417, 731

Miroshnichenko, A. S., Manset, N., Zharikov, S. V., et al. 2014, Adv. Astron., 2014, E7

Monnier, J. D., Millan-Gabet, R., Billmeier, R., et al. 2005, ApJ, 624, 832

Montesinos, B., Eiroa, C., Mora, A., \& Merín, B. 2009, A\&A, 495, 901

Morrell, N., \& Levato, H. 1991, ApJS, 75, 965

Mottram, J. C., Vink, J. S., Oudmaijer, R. D., \& Patel, M. 2007, MNRAS, 377, 1363

Nakano, M., Sugitani, K., Watanabe, M., et al. 2012, AJ, 143, 61

Natta, A., \& Whitney, B. A. 2000, A\&A, 364, 633

Natta, A., Grinin, V. P., Mannings, V., \& Ungerechts, H. 1997, ApJ, 491, 885

Oudmaijer, R. D. 2017, The B[e] Phenomenon: Forty Years of Studies, 508, 175

Oudmaijer, R. D., \& Drew, J. E. 1997, A\&A, 318, 198

Oudmaijer, R. D., \& Drew, J. E. 1999, MNRAS, 305, 166

Oudmaijer, R. D., Busfield, G., \& Drew, J. E. 1997, MNRAS, 291, 797

Oudmaijer, R. D., Proga, D., Drew, J. E., et al. 1998, MNRAS, 300, 170

Oudmaijer, R. D., Palacios, J., Eiroa, C., et al. 2001, A\&A, 379, 564

Patel, P., Sigut, T. A. A., \& Landstreet, J. D. 2017, ApJ, 836, 214

Pecaut, M. J., \& Mamajek, E. E. 2013, ApJS, 208, 9
Pogodin, M. A., Hubrig, S., Yudin, R. V., et al. 2012, Astron. Nachr., 333, 594

Polster, J., Korčáková, D., Votruba, V., et al. 2012, A\&A, 542, A57

Pomohaci, R., Oudmaijer, R. D., Lumsden, S. L., Hoare, M. G., \& Mendigutía, I. 2017, MNRAS, 472, 3624

Poxon, M. 2015, J. Am. Assoc. Var. Star Obs. (JAAVSO), 43, 35

Reiter, M., Calvet, N., Thanathibodee, T., et al. 2018, ApJ, 852, 5

Rivinius, T., Carciofi, A. C., \& Martayan, C. 2013, A\&ARv, 21, 69

Sartori, M. J., Gregorio-Hetem, J., Rodrigues, C. V., Hetem, A., Jr., \& Batalha, C. 2010, AJ, 139, 27

Schöller, M., Pogodin, M. A., Cahuasquí, J. A., et al. 2016, A\&A, 592, A50

Schütz, O., Meeus, G., Carmona, A., Juhász, A., \& Sterzik, M. F. 2011, A\&A, 533, A54

Skrutskie, M. F., Cutri, R. M., Stiening, R., et al. 2006, AJ, 131, 1163

Spezzi, L., Alcalá, J. M., Covino, E., et al. 2008, ApJ, 680, 1295

Tambovtseva, L. V., Grinin, V. P., \& Weigelt, G. 2014, A\&A, 562, A104

Taylor, M. B. 2005, Astronomical Data Analysis Software and Systems XIV, 347, 29

Testi, L., Palla, F., \& Natta, A. 1999, A\&A, 342, 515

Thé, P. S., de Winter, D., \& Perez, M. R. 1994, A\&AS, 104, 315

Torres, C. A. O., Quast, G. R., da Silva, L., \& de La Reza, R. 2000, IAU Symp., 200, 118

van den Ancker, M. E., The, P. S., Feinstein, A., et al. 1997, A\&AS, 123, 63

van den Ancker, M. E., de Winter, D., \& Tjin A Djie, H. R. E. 1998, A\&A, 330, 145

Vieira, S. L. A., Corradi, W. J. B., Alencar, S. H. P., et al. 2003, AJ, 126, 2971

Vieira, R. G., Gregorio-Hetem, J., Hetem, A., et al. 2011, A\&A, 526, A24

Vink, J. S., Drew, J. E., Harries, T. J., \& Oudmaijer, R. D. 2002, MNRAS, 337, 356

Waters, L. B. F. M., Cote, J., \& Lamers, H. J. G. L. M. 1987, A\&A, 185, 206

Watson, C. L., Henden, A. A., \& Price, A. 2006, Society for Astronomical Sciences Annual Symposium, 25, 47

Wheelwright, H. E., Oudmaijer, R. D., \& Goodwin, S. P. 2010, MNRAS, 401, 1199

Wheelwright, H. E., Vink, J. S., Oudmaijer, R. D., \& Drew, J. E. 2011, A\&A, 532, A28

Zhang, P., Yang, H. T., \& Liu, J. 2006, Ap\&SS, 305, 11

Zhang, Y., Tan, J. C., \& Hosokawa, T. 2014, ApJ, 788, 166

Zhang, Q., Claus, B., Watson, L., et al. 2017, ApJ, 837, 53

Zuckerman, B., Melis, C., Song, I., et al. 2008, ApJ, 683, 1085 